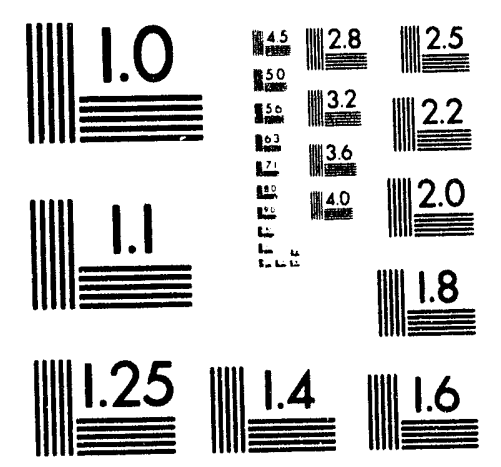



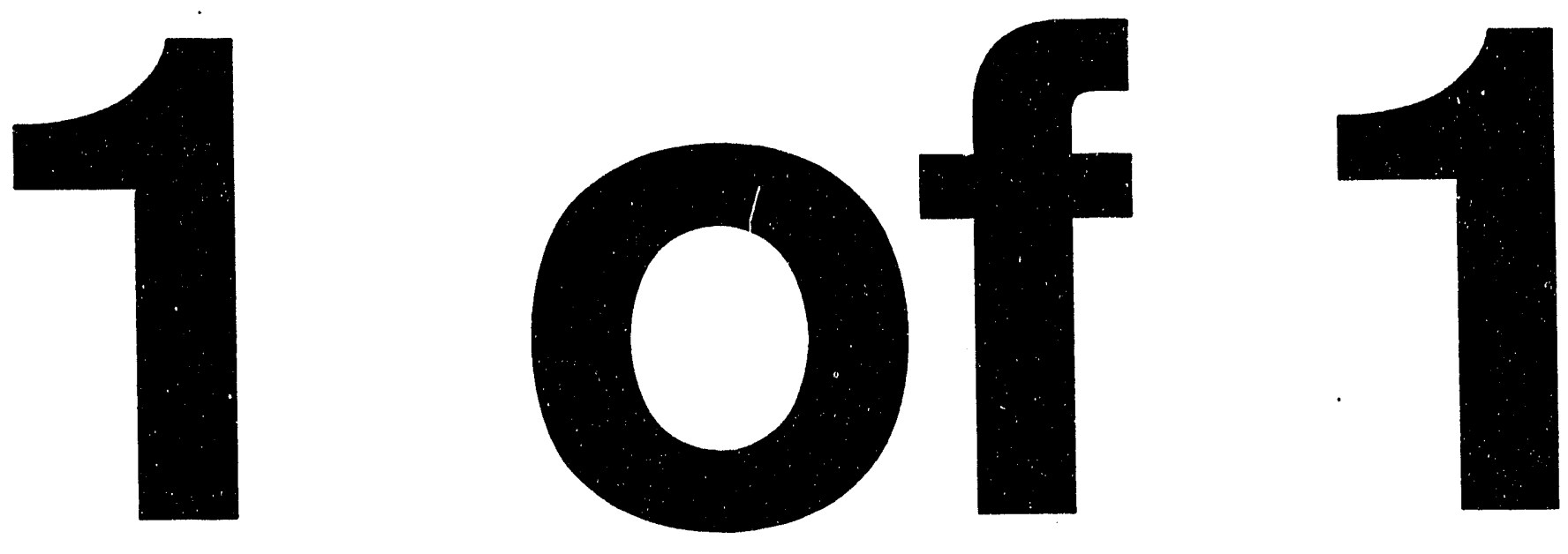


\title{
Studies of Global Energy Confinement in TFTR Supershots
}

\author{
J. D. Strachan \\ Plasma Physics Laboratory, Princeton University \\ P.O. Box 451 \\ Princeton, NJ 08543 USA
}

The global energy confinement time, $\tau_{E}$, from TFTR supershot plasmas has been correlated with the hydrogen recycling and the pressure anisotropy. An expression for the global confinement was obtained that describes its value at the time of peak neutron emission for all TFTR supershots obtained in the 1990 campaign, and simultaneously describes the time evolution of $\tau_{E}$ for an extensive subset of the 1990 data. The obtained expression is probably not unique and it can be written with different variables. An analysis of the energy balance for many of these supershots indicates that the primary effect of larger $\tau_{E}$ is that the central particle diffusivity is lower. 


\section{Introduction}

This paper describes one set of plasma and machine parameters which correlate with the global energy confinement time of TFTR supershot plasmas. The motivation is that knowledge of the parameters which correlate with plasma confinement may help identify underlying physical processes which control transport. The present work takes the perspective of attempting to simultaneously characterize the global energy confinement time near peak energy as well as its time evolution. The principal new result is that a dependence upon pressure anisotropy and hydrogen recycling is evident.

Previous publications have shown that supershot plasmas on TFTR exhibit a scaling [1] of

$$
\tau_{E} \propto F_{n}
$$

with no dependence upon beam power and plasma current. $F_{n}$ is the density peakedness $\equiv$ $\left.\mathrm{n}_{e}(0) /<\mathrm{n}_{e}\right\rangle$, and $\tau_{E}$ is the global energy confinement time measured magnetically [2].

The empirical scaling in Eq. (1) also applies to the supershot data set used in this paper (Sec. 2) (Fig. 1). However, Eq. (1) has some deficiencies. Firstly, there is a lot of scatter in Fig. I so that there can be a wide range of confinement times at a given density peakedness or a wide range of $F_{n}$ at a given $\tau_{E}$ (Fig. 1). Secondly, $\tau_{E}$ saturates at high density peakedness such that there is no dependence of $\tau_{E}$ on $F_{n}$ for $F_{n}>2.5$. Thirdly, (Sec. 3.2), the time evolution of $\tau_{E}$ and $F_{n}$ are usually uncorrelated through a supershot (e.g., Fig. 2), so that Eq. (1) seems to be valid only when relating different plasmas near times of peak energy content. Finally, when Eq. (1) has been used as the motivation for techniques to improve confinement, then the improved confinement has often not occurred (e.g., deuterium pellet studies [3] which are discussed later). Thus, Eq. (1) does not contain all of the important 
parameters that describe supershot energy confinement.

Recently, a different TFTR supershot scaling for the energy confinement time at peak neutron emission was found [4]

$$
\tau_{E} \propto B^{1.07} I_{p}^{0.22} W_{B}^{-0.56} C_{N B}^{0.30} C I I^{-0.32}
$$

$B$ is the toroidal magnetic field, $I_{p}$ is the plasma current, $W_{B}$ is the beam voltage, $C_{N B}$ is $\frac{P_{r o}}{P_{B}}$, and $\mathrm{CII}$ is the CII light in the ohmic plasma just prior to the application of the neutral beam heating. This scaling reflects the operational efforts on TFTR to improve the supershot plasma which principally are attempts to improve the plasma wall conditioning and. thus reduce the CII light in the ohmic plasma prior to beam heating. Equation (2) has all of its parameters fixed in time and, therefore, also cannot follow the time evolution, $\tau_{E}(t)$.

The purpose of this paper was to find a scaling relation that will describe the TFTR supershot energy confinernent both at time of peak neutron emission (i.e., near peak energy content) as well as for the time evolution. The organization of this paper is that Sec. 2 describes the TFTR dat,a that has been studied and the variables that were chosen for the analysis. The analysis indicates that several scalings are possible depending upon which data are emphasized in the analysis. Section 3 describes the scaling proposed in this paper. Section 4 examines the ability of this scaling to fit supershot $\tau_{E}$ at the peak neutron emission time. Sections 5 describes the ability of the equation to fit the time evolution of supershots. Section 6 relates the measured global confinement time to the transport coefficients deduced from an energy balance. 


\section{TFTR Plasmas}

The approach taken in this paper has been to examine all of the supershot plasmas that were obtained during the extensive 1990 run campaign [4]. The data are intended to encompass the full range of plasma behavior encountered in 1990 when TFTR was operated in the supershot mode [5]. The plasmas used in this study all rise from the 1990 experiments aimed towards improving the fusion vield in TFTR supershots. The main restrictions on the data set are that the plasmas: (1) be free of neutral beam faults, (2) be well diagnosed. (3) have $2.45 \mathrm{~m}$ major radius, and (4) have $\tau_{E}>1.50 \tau_{E}^{L}$ (L-mode confinement) at the time of peak neutron emission. The 1990 campaign consisted of 41 run days during which approximately 2800 plasma shots were run. These shots consisted of approximately 1500 helium conditioning shots, 1125 neutral beam heating shots, and 100 ohmic shots. At that time, it was found that $\mathrm{R}=2.45 \mathrm{~m}(\mathrm{a}=80 \mathrm{~m})$ plasmas had the largest neutron emissions and so many of the plasmas were run at $R=2.45 \mathrm{~m}$. If we consider only those plasmas at $\mathrm{R}=2.45 \mathrm{~m}$, then there were 525 well diagnosed, fault-free supershot plasmas which are all included in this study. There were no current ramps, RF power, or major radius excursions included in the present data set. The restriction of the energy confinement to being $50 \%$ larger than L-mode confinement eliminates only a few plasmas and attempts to avoid L-mode plasma behavior.

The 1990 campaign had three separate phases: (1) firstly, the emphasis was upon limite: conditioning [6] following the vacuum opening and evaluation of the new inner wall bumper limiter [T], (2) secondly, options were tried in order to optimize supershots. and (3) finally, the neutral beam power was maximized and injected into near optimum supershot target plasmas. The effort to run TFTR at maximum neutral beam power into a near optimized 
supershot target plasma resulted in 114 plasmas that nad greater than $30 \mathrm{MW}$ of neutral beam power. In order to avoid plasmas limited by the global $\beta$ limit, the plasma current was increased (up to 2 MA) in order to keep the plasma energy content below 0.8 of the Trovon beta limit $\left(2.3 \mathrm{IB} / \mu_{\circ} \mathrm{a}\right)$. There were only tw'o major disruptions at high power, and data from the disruption plasmas are not included in this study. Since the available beam power was higher and the approach with respect to disruptions was more conservative than previous runs, the plasma current tended to be a higher for the 1990 campaign. This range of plasma currents has little consequence except that few 1990 supershots were run below 1.0 MA, and the few plasmas at $0.85 \mathrm{MA}$ (14 supershots) accually present the worst fit to the proposed scaling relation in this paper. Moreover, due to the sparsity of available 1990 data at $<1.0 \mathrm{MA}$, it proved difficult to alter the scaling relation to adequately encompass the lower current plasmas. This feature will be discussed more fully in Sec. 5, but for the moment it is enough to note that the proposed scaling relation applies for plasma currents, $I_{p} \geq 1.0 \mathrm{MA}$, and that significant differences occur at lower plasma currents.

There were several other changes in TFTR which impacted the 1990 data and also slightly set this data set apart from previous supershot data. These include:

(a) The performance of the neutral beam injectors was improved so that there was more $(\sim 10 \%)$ power obtained at a given voltage [8].

(b) The carbon blooms [9] were eliminated. Thus, by simultaneously keeping $3<0.8$ $\beta_{\text {Troyon }}$ and avoiding carbon blooms. the confinement characteristics were not influenced trivially by these limits. The carbon blooms were eliminated by more precise limiter alignment to distribute the heat load more uniformly and by constructing the carbon tiles from stronger forms of carbon [ 7$]$. While blooms did not influence con- 
finement during 1990, some sporadic small blooms did occur, and it was observed that these correlated with slightly improved confinement on the subsequent supershot [10]. Examples of plasmas experiencing a bloom and benefitting from a preceding bloom are both included in the present data set.

(c) Several vigorous attempts to improve the limiter conditions were undertaken. These met with various degrees of success and were reported in detail elsewhere [10]. However. the most successful of these was uhe application of a thin Li coating by Li pellet injection [11]. Plasmas improved by Li pellets are included in the present data set.

Two data sets have been created from the 1990 supershots. The first stored the global energy confinement time at peak neutron emission for all 525 supershot plasmas, and the second considered the time evolution of the global energy confinement of 22 supershots. These 22 supershots were chosen to be representative of the types of evolution that had occurred during the beam heating of supershots in 1990 (Tables 1 to $\tau$ ). These evolutions include plasmas without MHD or blooms (Tables 1 and 2), plasmas experiencing blooms (Table 3), plasmas experiencing MHD but not blooms (Table 4), plasmas benefitting from a preceding bloom (Table 5), plasmas benefitting from lithium pellet conditioning (Table $5)$, overdrive plasmas (Table 6), and plasmas with density peakedness enhanced by pellets (Table 7 ).

\section{Global Energy Confinement Scaling}

Each of the above data sets (Sec. 2) have been studied by regression analysis in order to find parameters which statistically correlate with the global energy confinement time. $A$ single scaling expression was not found that fit all the data. However, an expression was 
found that was a reasonable fit to most of the data.with specific limitations. That expression is

$$
\tau_{E}=i 3 H_{\alpha}^{-0.24}\left(\frac{\beta_{\| 1}}{\beta_{\perp}}\right)^{0.85} n_{e}(0)^{0.60} B^{0.35} C_{. N B}^{0.19},
$$

where $\tau_{E}$ is the global energy confinement time $=E /\left(P_{B}-d E / d t\right), E$ is the magnetically measured total energy content, and $P_{B}$ is the neutral beam power. $H_{\alpha}$ is the summed $H_{\alpha}$ light emission from five vertically viewing chords [12] and is thought to be due to recycled deuterium for the plasmas discussed here. $\beta_{\|} / \beta_{\perp}$ is the pressure anisotropy determined from the diamagnetic and equilibrium energy contents, $n_{e}(0)$ is the central electron density, $B$ is the toroidal magnetic field, and $C_{N B}$ is the beam sense $-\mathrm{P}_{c 0} / \mathrm{P}_{B}$ where $\mathrm{P}_{c o}$ is the beam power injected parallel to the plasma current. This expression is derived from a composition of several regression analyses. The $\mathrm{H}_{\alpha}$ term arises primarily from the time evolution of $\tau_{E}(\mathrm{t})$ in supershots while $\beta_{\|} / \beta_{\perp}$ arises from the overdrive experiments, and the remaining terms are predominated in the data set from time of peak neutron emission. The evidence for the existence of each dependence will be presented in the next sections.

There are several specific limitations to the application of Eq. (3):

1. It applies only for supershots with plasma current $\geq 1.0 \mathrm{MA}$. This limitation arises since only a small subset of the 1990 supershot plasmas were at $0.85 \mathrm{MA}$. and these plasmas have a $\tau_{E}$ which is about 1.5 times higher than Eq. (3). Interestingly, the time evolution and scaling of just these 0.85 MA supershots follows the dependencies of Eq. (3) but the numerical factor is apparently different than for plasmas with $I_{p} \geq$ 1.0 MA.

2. The $\mathrm{H}_{\alpha}$ variation arises from the time evolution of $\tau_{E}(\mathrm{t})$; however, when it is applied to the data taken at the time of peak neutron emission, then there is a residual variation 
in $\tau_{E}$. It is as if the $\mathrm{H}_{\alpha}$ dependence applies strongly on the time evolution of a single plasma but is not as useful in relating different plasmas. Such a situation might occur if the physical meaning of $\mathrm{H}_{\alpha}$ were to change significantly between shots (for example. due to a evolving calibration or limiter-contact interaction). This situation might also occur if $\mathrm{H}_{\alpha}$ were related to but not exactly the correct variable to be used in Eq. (3). Further, it is possible that the $\mathrm{H}_{\alpha}$ variable in Eq. (3) is the origin of the difficulty with the 0.85 MA plasmas (above) since $\mathrm{H}_{\alpha}$ changes by greater than a factor-of-two between $0.85 \mathrm{MA}$ and the plasma currents above 1.0 MA.

3. The set of parameters used in Eq. (3) are not unique. Statistically, in the present data sets, several of these parameters are related to other parameters which could have been used nearly as well. For example, $\beta_{\|} / \beta_{\perp}$ is coupled to $n_{e}(0)$ and $H(0)$ the neutral beam deposition on axis and $\mathrm{H}_{\alpha}$ is related to the edge electron density. A relation which is almost as good as Eq. (3) is

$$
\tau_{E} \propto H_{\alpha}^{-0.24} n_{e}^{0.52}(o) H^{0.20}(o) B^{0.29} C_{N B}^{0.19}
$$

In this study, we were unable to completely explore the full range of possible scaling relations and instead feel forced to admit this limitation and proceed anyhow to examine the quality of the correlation of Eq. (3) with the TFTR 1990 data. On the other hand, the parameters used in Eqs. (3) and (4) do represent the areas of physics that seem involved with supershot confinement, namely, the plasma edge $\left(\mathrm{H}_{\alpha}\right)$; the density peakedness $\left(n_{e}(0)\right)$; the use of neutral beam heating $\left(H(0)\right.$ or $\beta_{\|} / \beta_{\perp}$, and $\left.C_{N B}\right)$; and high toroidal magnetic fields (B).

4. The coefficients in Eqs. (3) and (4) are quoted without uncertainty limits. We were unable to establish that these coefficients are unique or even that a power law 
dependence is appropriate. Instead. the benefit of the coefficients quoted in Eq. (3) and $(4)$ is that factors are identified which relate to improving confinement.

5. The meaning of the global energy confinement time can differ throughout the evolution of the supershot. Higher $\tau_{E}$ can represent higher $\frac{d E}{d t}$, beam energy content. lower convection. or lower conduction coefficients. Thus, it is possible that some dependencies in Eqs. (3) and (4) apply to only one physical process which may not describe all supershot conditions. In this sense, transport analyses which derive transport coefficients are preferable. The two major difficulties with such transport analysis are: (1) the requirements of more extensive diagnositc coverage reduce the number of plasmas that are studied, and (2) the statistical scatter that arises from analyzing gradients can be large. Our attempts at transport analysis are described in Sec. 6 . The benefit of the global energy confinement time is that it is measured accurately on all the 1990 supershot data and, therefore, can encompass more data than the transport analysis. The study in Sec. 6 indicates that improved global $\tau_{E}$ correlates primarily with improved particle transport.

6. Finally, it is a limitation that Eqs. (3) and (4) are not derived rigorously from one data set. This means that Eq. (3) is not the best description of any one data set but is a compromise that works moverately well for almost all 1990 data.

\section{Description of $\tau_{E}$ at Time of Peak Neutron Emission}

The energy confinement time at peak neutron emission for all the 1990 supershot plasmas is reasonably described by Eq. (3) (Fig. 3) with a ratio of $1.00 \pm 0.07$. The nature of a large scatter plot with all the data on it is that the outlying data points tend to be emphasized 
(Fig. 3B) while binning the data together and averaging (Fig. 3A) tends to overemphasize the agreement of Eq. (3) with $\tau_{E}$. Probably, an accurate statement is that Eq. (3) describes almost all of the 1990 supershot global energy confinement times at peak neutron emission but that there are some plasmas with $20 \%$ deviations (approximately $2 \%$ of the data set).

Isolating different types of plasmas indicates that essentially all the supershot plasma attempts can be described by Eq. (3). In particular, supershot plasmas with sawtooth MHD activity are as well described as supershot plasmas without sawtooth MHD activity (Fig. 4). The two plasma attempts in 1990 with Co-only beam injection are as well described as are supershot plasmas with nearly balanced injection (Fig. 5). Supershot attempts which featured lower confinement (within $50 \%$ of L-mode) are as well described as plasmas with $\tau_{E}>1.5 \tau_{E}^{L}$ (Fig. 5). The supershot attempts that were aided by Lithium pellet conditioning or blooms on the preceding plasma are about as well described by Eq. (3) as are supershot not influenced by either (Fig. 6A). The supershots immediately (within 3 shots) following helium discharge cleaning sequences tended to be described by Eq. (3) but also account for the bulk of the plasmas below the unity line (Fig. 6B). The plasmas which followed boronization (within 30 shots. Fig. 6B), and the plasmas aided by Lithium pellets (Fig. 6A) were somewhat improved compared to Eq. (3) (about $6 \%$ each, on average).

The individual dependencies of the variables in Eq. (3) can be seen explicitly. In Fig. $\bar{i}$, $\tau_{E}$ increases with $n_{e}(0)$ when the other variables are kept constant for two different $\mathrm{H}_{\alpha}$ values. The 'ower $\mathrm{H}_{\alpha}$ value has a higher $\tau_{E}$ (Fig. 7 ). Also for the other parameters being constant, $T_{E}$ increases with $\hat{y}_{\|} / \beta_{\perp}$ (Fig. 8 ). The dependence on beam balance is probably a difference between Co- and Counter dominated beam injection (Fig. 9). The toroidal magnetic field dependence has about a $20 \%$ effect when going from $3.9 \mathrm{~T}$ to $5.2 \mathrm{~T}$ (Fig. 10). The variation in $\tau_{E}$ with $\beta_{\|} / \beta_{\perp}$ is the largest $(\sim 50 \%)$ while the remaining parameters produce $20 \%$ variations 
in $\tau_{E}$ for the 1990 supershot plasmas. There is some residual variation of $\tau_{E}$ with respect to Eq. (3) at large $\mathrm{H}_{\alpha}$ levels (Fig. 11). The $\mathrm{H}_{\alpha}$ coefficient in Eq. (3) was determined primarily by the time evolution data, and thus, Fig. 11 indicates that the $\mathrm{H}_{\alpha}$ dependence in Eq. (11) does not connect well to plasmas with large $\mathrm{H}_{\alpha}$ levels. Equation (3) seems to describe supershot attempts that are close to L-mode as well as supershot attempts that are 2.5-3 times L-mode (Fig. 12).

\section{Time Evolution of Supershots}

The time evolution of all the 1990 supershots was observed and categorized into the groups of Tables 1-7. Although only 22 of the 525 supershots are described in this section, these plasmas are representative of the range of time evolution of 1990 supershot plasmas. In all cases, the neutral beam heating power was fault free. The data before $0.2 \mathrm{sec}$ of beam heating was not used due to potentially larger beam shinethrough.

\subsection{Time Evolution of Supershots Free of MHD or Blooms}

The evolution of the supershot plasma is characterized by a peaking of the stored energy and neutron emission at about $0.5 \mathrm{sec}$ into the beam heating. The decay of the stored energy and neutron emission has often been ascribed to the effect of MHD [13] and/or blooms [14] on the plasma. In 1990, there were four (4) plasmas where the decay of the neutrons and the stored energy could not be ascribed to either blooms or the MHD activity (as observed on the Mirnov coils). These plasmas (Tables 1 and 2) included lower power beam heating (Table 1) where two of the plasmas had essentially constant confinement time such that the neutron emission and stored energy did not decay (Figs. 13 and 14). The higher power plasmas 
(Table 2) which were MHD and carbon bloom free'(Fig. 15) featured a distinct confinement time degradation which resulted in the neutron emission and the energy content decaying. The plasmas exhibit a reasonable (but not perfect) agreement with the $\tau_{E}$ value for these plasmas (Fig. 16).

\subsection{Time Evolution of Supershnts Experiencing a Carbon Blooms}

The time evolution of a series of plasmas which experie sce a carbon bloom (Table 3) coincident with a degradation in $\tau_{E}$ indicates that the parameters that have correlated with $T_{E}$ in plasmas without blooms or strong MHD (Sec. 5.1) also are varying (Fig. 17). The plasmas in Tables 3 are intended to span the range in magnitude and time evolution of the degradation in $\tau_{E}$ that occurred in 1990 supershots coincident with blooms. The strongest correlator of the $\tau_{E}$ degradation in the bloom is with the increased $\mathrm{H}_{\alpha}$ signal (Figs. 17,18) that occurred at the bloom. Apparently, the bloom is associated with the release of deuterium from the limiter. The transition of the confinement from supershot values all the way down to L-mode values is described by Eq. (3) (Fig. 19).

\subsection{Time Evolution of Supershots Experiencing Large MHD}

Often the time evolution of $\tau_{E}$ in supershot plasmas degrades coincident. with the onset of continuous MHD (typically but not exclusively $3 / 2$ modes). Three plasmas have been considered (Table 4) which have varying severity of $\tau_{E}$ degradation at the onset of the strong MHD. In each case. Eq. (3) reasonably describes the confinement degradation (Figs. 20.21) with the time evolution of the central density and $\mathrm{H}_{\alpha}$ signals being the primary correlations with the $\tau_{E}$ degradation. The evolution of these plasmas and those degraded coincident 
with blooms all seem reasonably described by Eq. (3) (Fig. 19). As is evident from Figs. 13 - 19. the $\mathrm{H}_{\alpha}$ signal is a reasonable inverse correlation with $\tau_{E}$. In fact, the individual plasmas can be overlayed (Fig. 22) to yield a reasonable inverse dependence on $\mathrm{H}_{\alpha}$. It is the single strongest variation evident for the time evolution of the degradation in $\tau_{E}$.

\subsection{Supershot Plasmas Improved by Use of Lithium Pellets}

We now consider several plasmas improved by a preceding bloom [10] or a preceding lithium pellet [11] with some comparison supershot plasmas (without a preceding bloom nor a lithium pellet) (Table 5). The evolution of all of these shots featured a decaying $\tau_{E}(t)$ that was described by Eq. (3) (Figs. 23-25). The enhancement in confinement correlated with the $\mathrm{Li}$ pellet or preceding carbon bloom is attributed (by Eq. (3)) to be due to a simultaneous increase in the central density and a decrease in $\mathrm{H}_{\alpha}$ (Figs. 24,25). Again, Eq. (3) had more difficulty describing the lower plasma current case (Fig. 25) especially early in the beam heating.

\subsection{Overdrive Plasmas}

During the 1990 run. experiments were performed with a time-evolving beam power [15]. Since the supershot plasma seems to require central fueling by energetic ions, experiments were performed to enhance the fueling. A short initial burst of high power was applied and the power levels was subsequently reduced. and the plasma was overdriven with beam power for $0.1 \rightarrow 0.15 \mathrm{sec}$. Usually, the plasma current was not sufficient to sustain the plasma energy content if the initial power were applied for more than about one confinement time since the plasma would approach the Trovon Stability Limit. Thus, these overdrive plasmas 
evolved with more beam fueling in the first $\tau_{E}$ than would normally occur in supershot plasmas.

The evolution of some plasmas did have a persistent slight improvement in energy content. $T_{E}$, neutron emission, and density peakedness compared to similar shots without the overdrive power (Fig. 26). The improvement persisted for several energy conficement times. Some of these plasmas had the highest density peakedness that have so far been attained in a supershot plasma. For the shot pair in Fig. 26 and Table 6, the target CII light and target density were a little higher for the overdrive plasma, and the edge parameters (CII, $\mathrm{H}_{\alpha}$, and edge density) were higher for the overdrive shot. The overdrive plasmas are interesting since $\mathrm{H}_{\alpha}$ and $\mathrm{n}_{e d g e}$ are higher with the higher confinement time and the overdrive. According to Eq. (3), the overdrive plasma had higher confinement times primarily because they had higher central densities and higher pressure anisotropy (Fig. 27). The plasmas in Table 6 and Fig. 27 have lower plasma currents than for the remainder of the supers'ots discussed in this paper (0.8 MA) and are deviant from Eq. (3) due to this reason. There were some overdrive attempts at 1.4 MA and these (and their comparison plasmas) are well described by Eq. (3) (Fig. 28).

\subsection{Comparison Between Deuterium Pellet Plasma and Supershot}

One consequence of a scaling law is that it suggests methods for improving $\tau_{E}$. For example, Eq. (1) suggests that deuterium pellet fueling of supershot plasmas might further enhance the density peakedness and, therefore, $\tau_{E}$. However, such attempts actually degraded the confinement (although $\tau_{E}$ was still higher than L-mode) [3].

An interesting shot pair is the neutral beam heated deuterium pellet fueled plasma in 
Table 7 compared to a supershot with similar densily peakedness (Fig. 29). The deuterium recycling and the pressure anisotropy are both different for these plasmas in a manner that allows Eq. (3) to describe $\tau_{E}$ (Fig. 29).

\section{Discussion}

The regression analysis of the 1990 supershots indicates that a number of fits to the confinement time data are possible. Of these expressions, Eq. (3) has the widest application. It reasonably approximates the time evolution of the energy confinement time over the full range of supershot plasma behavior observed on TFTR during 1990 (for $R=2.45 \mathrm{~m}$ ). However, the agreement is not perfect. The differences between $\tau_{E}$ and Eq. (3) can be large and are typically $\approx 10 \mathrm{msec}$. It is worth noting that the 1990 experimental effort was often centered around attempting to make $10 \mathrm{msec}$ improvements in $\tau_{E}$. Further, the low current plasmas $\left(I_{p} \sim 0.85 \mathrm{MA}\right)$ are not well described by Eq. (3), so that it is limited in its utility to the range $1 \rightarrow 2$ MA where most of the 1990 supershot data was taken. It is possible that the $\mathrm{H}_{\alpha}$ dependence in $\mathrm{Eq}$. (3) is sensitive to the plasma current since the edge signals $\mathrm{n}_{\text {edge }}, \mathrm{CII}$, and $\mathrm{H}_{\alpha}$ are proportional to $\mathrm{I}_{p}$ at low beam powers.

With Eq. (3) approximately able to describe $\tau_{E}$, the principal parameter involved in improving or degrading confinement can be identified for the 1990 supershot experiments. Improvements to $\tau_{E}$ caused by lithium pellet conditioning are interpreted by Eq. (3) to be due to a combination of higher central densities and lower $\mathrm{H}_{\alpha}$ light emission. Improvements caused by the overdrive experiments or by the occurrence of a bloom on the preceding plasma are due to higher central densities. Both supershot plasmas that have sawtooth MHD activity and those which do not tend to have $\tau_{E}$ which scales with Eq. (3). The 
scaling indicates that sawtooth plasmas have lower central densities and thus lower $\tau_{E}$. A few plasmas had $\mathrm{C}_{N B}=1$ (i.e., Co only beam injection) and for these $\tau_{E}$ was described by Eq. (3). The Co only plasma extend the $C_{N B}^{19}$ scaling of Eq. (3) but tended to have low central densities.

The degradation of ${ }^{\circ} E$ during the time evolution of the MHD free and bloom free plasmas is attributed to an increase in $\mathrm{H}_{\alpha}$ and a decrease in $\beta_{\|} / \beta_{\perp}$. The degradation correlated with a carbon bloom was primarily associated with an increase in $\mathrm{H}_{c}$, and, secondarily, by a decrease in $\beta_{\|} / \beta_{\perp}$. These plasmas degraded nearly to $L$-mode indicating that the $\mathrm{H}_{\alpha}$ magnitude is also different between supershots and L-mode. The degradation in $\tau_{E}$ correlated with an increase in MHD activity is primarily associated with an increase in $\mathrm{H}_{\alpha}$ and a secondary decrease in $\beta_{\|} / \beta_{\perp}$. The deuterium pellet plasma had lower $\tau_{E}$ since it had significantly lower $\beta_{\|} / \beta_{\perp}$ than the supershot at the same density peakedness.

\section{Steady-State Transport Calculations}

For 118 of the 1990 supershots, a SNAP analysis [16] was performed of the one-dimensional energy balance to deduce $\chi_{i}(r), \chi_{e}(r)$, and $D_{e}(r)$. The principal assumptions used in the analysis are that steady-state conditions exist for the plasma characterized by the measurements of $T_{e}(r), T_{i}(r), \beta_{\text {equil }}, \beta_{\text {diam }}, Z_{\text {metals }}$ and $\mathrm{Z}$ from visible bremsstrahlung (presumed flat). For these cases. the calculated neutron emission and energy content agreed with the measurements. $\left(\mathrm{I}_{n S N A P} / \mathrm{I}_{n}=0.90 \pm 0.10\right.$ and $\left.\mathrm{E}_{S N A P} / \mathrm{E}=0.99 \pm 0.04\right)[4]$ indicating that the plasma measurements were all consistent with each other.

To identify the location of changes in the transport coefficients when $\tau_{E}$ has changed, the data was separated into two sets. The first set is of the lowest $\tau_{E}$ values that were analyzed 
(16 plasmas with an average $\tau_{E}=0.116 \mathrm{sec}$ ). . The second set is the highest $\tau_{E}$ values that were analyzed ( 5 plasmas with an average $\tau_{E}=0.162 \mathrm{sec}$ ). Both groups of plasmas are supershot plasmas. The improvement in confinement time (Fig. 30) correlated with a central $(\mathrm{r} \leq 30 \mathrm{~cm})$ reduction and an outer $(\mathrm{r}>50 \mathrm{~cm})$ more modest increase in $\chi_{i}$ and $\mathrm{D}_{\varrho}$. $\chi_{e}$ (total of conduction and convection losses analyzed as entirely due to conduction) was relatively unchanged with modest changes occurring at $r \sim 40 \rightarrow 50 \mathrm{~cm}$. The variation of the global $\tau_{E}$ is correlated primarily with changes in the central thermal plasma transport coefficients $\gamma_{i}$ and $D_{e}$. These two parameters are inter-related in the analysis since the convection term has been subtracted from the power to the ions to infer $\chi_{i}$. An assumed convection multiplier of $3 / 2$ was used, and it is quite possible that the convection multiplier should actually be a different value so that $\chi_{i}$ might not be well-determined by these SNAP calculations.

Following the ideas of Scott [17], $\mathrm{D}_{e}(40 \mathrm{~cm})$ and $\mathrm{D}_{e}(60 \mathrm{~cm})$ do decrease when the Lmode scaling of $\tau_{E}$ increased, while $\mathrm{D}_{e}(25 \mathrm{~cm})$ was relatively unchanged (Fig. 31). Possibly L-mode and supershots act upon different parts of the plasma with the L-mode describing the outer particle and energy balance while supershots describe the central particle and possibly ion energy balance.

Direct regression was attempted upon the transport coefficients at $25 \mathrm{~cm}, 40 \mathrm{~cm}$, and $60 \mathrm{~cm}$. The strongest dependence was for $D_{e}(40 \mathrm{~cm})$. The plasma diffusivity, $D_{e}(40 \mathrm{~cm})$, regressed to

$$
D_{e}(40 \mathrm{~cm})=P_{B}^{1.02 \pm 0.06} I_{p}^{-1.14 \pm 0.14}
$$

where each dependence can be seen when the other parameter is constant (Fig. 32). Some of the scatter in the fit arises from the plasmas influenced by conditioning from preceding 
blooms and lithium pellets. Apparently, these wall interactions influence the plasma transport at the half-radius directly and not through the edge transport coefficients even though parameters such as the edge density and the $\mathrm{H}_{\alpha}$ light are changed.

\section{Summary}

This paper has statistically evaluated correlations of the TFTR supershot global energy confinement time with plasma parameters. It was found that scaling $\tau_{E}$ as $\mathrm{H}_{\alpha}^{-0.24} \beta_{\|} / \beta_{\perp}^{0.85}$ $\mathrm{n}_{e}^{0.6} \mathrm{~B}^{0.35} \mathrm{C}_{N B}^{0.19}$ can describe the entire variety of TFTR supershot conditions. The transport coefficient changes that correlate with the better supershots (higher $\tau_{E}$ ) are central reduction in particle diffusivity and, possibly, ion thermal conduction. Several different scalings for the global energy confinement time are possible. Some parameters that may alternately be important are the edge density and the peakedness of the beam deposition.

The main value in identifying these significant plasma parameters is to suggest possible physical mechanisms which might be determining plasma confinement. Certainly, the dependences of $\tau_{E}$ upon $\mathrm{H}(0), \mathrm{H}_{\alpha}$, and $\mathrm{n}_{e}(0)$ as well as the correlation of $\tau_{E}$ with reduced central $\mathrm{D}_{e}$ may indicate a possible connection between convection and $\tau_{E}$. The dependence upon pressure anisotropy does not suggest a correlation with beam ion density or energy content even through the pressure anisotropy does arise from the thermalizing beam ions since the beam ion density does not correlate with $\beta_{\|} / \beta_{\perp}$. The dependences of $\tau_{E}$ upon $\beta_{\|} / \beta_{\perp}, \mathrm{B}$, and $\mathrm{P}_{c o} / P_{B}$ may indicate a possible connection with stability and $\tau_{E}$. 


\section{Acknowledgment}

This work was supported by the U.S. Department of Energy Contract No. DE-AC02-76CHO-3073 


\section{References}

[1] PARK, H., Plasma Phys. Contr. Fusion 31 (1989) 2035.

[2] BELL, M.., et al., Plasma Phys. Contr. Fusion 28 (1986) 1329.

[3] PARK, H., et al., Bull. Am. Phys. Soc. 35, 2086 (1990).

[4] STRACHAN, J.D.. et al., "Neutron Emission from TFTR Supershots," Princeton Plasma Physics Report No. PPPL-2858 (1992).

[5] STRACHAN, J.D.. et al., Phys. Rev. Lett. 58 (1987) 1004.

[6] MUEller, D., et al., Princeton Plasma Physics Laboratory Report No. PPPL-2758 (1991)

[7] BARNES, G.W., et al., in Proc. of IEEE Conference (1991) to be published.

[8] KAMPerschroer, J., et al., Princeton Plasma Physics Laboratory Report No. PPPL-2763 (1991).

[9] RAMSEY, A., Princeton Plasma Physics Laboratory Report No. PPPL-2701 (1990).

[10] STRACHAN, J.D., et al., in Proc. 10th Int. PSI Conference (1992), (Monterey, CA.. 1992), to be published in J. Nucl Mater., Princeton Plasma Physics Laboratory Report No. PPPL-2841 (1992).

[11] SNIPES, J., et al., European Cont. on Plasma Phys. Contr. Fus., (Berlin, 1991).

[12] RAMSEY, A.T., and TURNER, S.L., Rev. Sci. Instrum. 58 (1987) 1211.

[13] MC GUIRE, K., et al., Pl. Phys. Contr. Nuci. Fus., Vol. I (1987) 421. 
[14] MEADE, D., et al.. in Proc. 13th Int. Conf. on Plas. Phys. and Contr. Nucl. Fus. Research (Washington, 1990) Vol. 1, pg. 9.

[15] BELL, .M., et al.. Bull. Am. Phys. Soc. 35, 2107 (1990).

[16] MURPhy, J.A., SCOTT, S.D., and TOWNER, H.H., Princeton Plasma Physics Laboratory Report No. PPPL-TM-393 (1992).

[17] SCOTT. S., et al.. in Proc. 13th Int. Conf. on Plasma Phys. Contr. Nucl. Fusion Research (1990), (Washington, DC, 1990). IAEA-CN-53/A-3-6. 
Table 1. Low Power :MHD Free Plasmas

\begin{tabular}{|c|c|c|c|c|c|c|c|}
\hline SHOT & $\mathrm{P}_{B}(\mathrm{MW})$ & $\mathrm{I}_{p}(\mathrm{MA})$ & $\mathrm{COFR}$ & $\mathrm{W}_{B}(\mathrm{kV})$ & $\mathrm{B}(\mathrm{T})$ & $\hat{\tau}_{E}(\mathrm{sec})$ & Comments \\
\hline 49928 & 12 & 1 & 0.21 & 93 & 4.7 & 0.17 & \\
52950 & 11.5 & 1.05 & -0.24 & 96 & 4.7 & 0.137 & \\
52969 & 11.5 & 1.05 & -0.21 & 96 & 4.7 & 0.135 & $\tau_{E}$ constant in time \\
52990 & 1.3 .5 & 1.05 & -0.02 & 96 & 4.7 & 0.158 & small bloom \\
\hline
\end{tabular}


Table 2. High Power MHD Free and Bloom Free Plasmas

\begin{tabular}{|c|c|c|c|c|c|c|c|}
\hline SHOT & $\mathrm{P}_{B}(\mathrm{MW})$ & $\mathrm{I}_{p}(\mathrm{MA})$ & $\mathrm{COFR}$ & $\mathrm{W}_{B}(\mathrm{kV})$ & $\mathrm{B}(\mathrm{T})$ & $\hat{\tau}_{E}(\mathrm{sec})$ & Comments \\
\hline 51354 & 30 & 1.8 & -0.14 & 104 & 5.2 & 0.125 & Small bloom \\
55848 & 25.5 & 1.6 & 0.23 & 106 & 5.1 & 0.16 & Li pellet. small $\mathrm{I}_{p}$ ramp \\
\hline
\end{tabular}


Table 3. Plasmas Experiencing Blooms

\begin{tabular}{|c|c|c|c|c|c|c|}
\hline SHOT & $\mathrm{P}_{B}(\mathrm{MW})$ & $\mathrm{I}_{p}(\mathrm{MA})$ & COFR & $\mathrm{W}_{B}(\mathrm{kV})$ & $\mathrm{B}(\mathrm{T})$ & $\hat{\tau}_{E}(\mathrm{sec})$ \\
\hline 47725 & 24 & 1.6 & 0.03 & 95 & 4.7 & 0.13 \\
51254 & 31 & 1.8 & -0.07 & 103 & 5.2 & 0.12 \\
51383 & 30.5 & 1.75 & -0.1 & 102 & 5.2 & 0.142 \\
55683 & 32 & 1.75 & 0 & 103 & 5.1 & - \\
\hline
\end{tabular}


Table 4. Plasmas Experiencing MHD

\begin{tabular}{|c|c|c|c|c|c|c|c|}
\hline SHOT & $\mathrm{P}_{B}(\mathrm{MW})$ & $\mathrm{I}_{p}(\mathrm{MA})$ & $\mathrm{COFR}$ & $\mathrm{W}_{B}(\mathrm{kV})$ & $\mathrm{B}(\mathrm{T})$ & $\hat{\tau}_{E}(\mathrm{sec})$ & Comments \\
\hline 48041 & 21 & 1.6 & 0.14 & 92 & 4.7 & 0.165 & \\
55850 & 25 & 1.6 & 0.13 & 106 & 5.1 & 0.175 & Li pellet, small $\mathrm{I}_{p}$ ramp \\
51267 & 31 & 1.75 & -0.07 & 103 & 5.2 & 0.147 & $\begin{array}{c}\text { benefitted from } \\
\text { (preceding bloom) }\end{array}$ \\
\hline
\end{tabular}


Table 5. Plasmas and Comparison Plasmas which are Influenced by a Lithium pellet or a Preceding Bloom

\begin{tabular}{|c|c|c|c|c|c|c|l|}
\hline SHOT & $\mathrm{P}_{B}(\mathrm{MW})$ & $\mathrm{I}_{p}(\mathrm{MA})$ & COFR & $W_{B}(\mathrm{kV})$ & $\mathrm{B}(\mathrm{T})$ & $\hat{\tau}_{E}$ & Comments \\
\hline 51550 & 27 & 1.6 & 0.3 & 101 & 5.2 & 0.14 & preceded by bloom \\
53782 & 23.5 & 1.7 & 0.13 & 103 & 5.1 & 0.13 & control for 53843 \\
53843 & 23.5 & 1.7 & 0.14 & 103 & 5.1 & 0.15 .5 & preceded by Li pellet \\
55005 & 20 & 1.4 & 0 & 104 & 5.1 & 0.13 & control for 55632 \\
55632 & 20 & 1.4 & 0 & 104 & 5.1 & 0.16 & preceded by Li pellet \\
\hline
\end{tabular}


Table 6. Overdrive Plasmas

\begin{tabular}{|c|c|c|c|c|c|c|c|}
\hline SHOT & $\mathrm{P}_{B}(\mathrm{MW})$ & $\mathrm{I}_{p}(\mathrm{MA})$ & $\mathrm{COFR}$ & $\mathrm{W}_{B}(\mathrm{kV})$ & $\mathrm{B}(\mathrm{T})$ & $\hat{\tau}_{E}(\mathrm{sec})$ & Comments \\
\hline 47789 & 10.5 & 0.8 & -0.005 & 91 & 4.9 & 0.16 & overdrive at $19 \mathrm{MW}$ for 0.2 sec \\
47791 & 10.5 & 0.8 & -0.005 & 91 & 4.9 & 0.13 & comparison shot \\
\hline
\end{tabular}


Table 7. Supershot Plasma Comparison to Deuterium Pellet

Fueled Plasma with the Same Density Peakedness

\begin{tabular}{|c|c|c|c|c|c|c|c|}
\hline SHOT & $\mathrm{P}_{B}(\mathrm{M} \mathrm{W})$ & $\mathrm{I}_{p}(\mathrm{MA})$ & $\mathrm{COFR}$ & $\mathrm{W}_{B}(\mathrm{kV})$ & $\mathrm{B}(\mathrm{T})$ & $\hat{\tau}_{E}(\mathrm{sec})$ & Comments \\
\hline 53848 & 26 & 1.7 & 0.22 & 102 & 5.09 & 0.16 & Comparison superhot \\
55053 & 23 & 2 & -0.12 & 101 & 5.1 & 0.12 & deuterium pellet \\
\hline
\end{tabular}




\section{Figures}

Fig. 1. The global energy confinement time, $\tau_{E}$, at the time of peak neutron emission plotted against the density peakedness, $F_{n}$, for all the 1990 supershot plasmas. (A) The data averaged over 20 bins with error bars being standard deviations of the mean. (B) All of the 1990 data. The closed circle is a supershot plasma attempt where large density peakedness was obtained by deuterium pellet injection into the target plasma [3] and the open circle is a comparison supershot plasma with the same density peakedness, plasma current, and beam power.

Fig. 2. Time evolution of (A) $\tau_{E},(B) F_{n},(C)$ total energy content, and (D) $H_{\alpha}$ signal (deuterium recycling) for a supershot plasma with 1.7 MA plasma current and $25 \mathrm{MW}$ beam power. Notice that $\tau_{E}$ is falling throughout the plasma evolution while $F_{n}$ is rising until $3.5 \mathrm{sec}$ and is constant thereafter.

Fig. 3. The measured global energy confinement time, $\tau_{E}$, plotted against Eq. (3) for all the 1990 supershot plasmas at the time of peak neutron emission: (A) the data grouped together into 15 bins with the error bars being the standard deviation of the mean, and (B) all the data.

Fig. 4. The measured global energy confinement time, $\tau_{E}$, plotted against.Eq. (3). The (X) data points do not have sawtooth MHD activity while the (口) data points do have sawtooth MHD activity.

Fig. 5. The measured global energy confinement time, $\tau_{E}$, plotted against Eq. (3). The (t) data points have $\tau_{E} \geq 1.5 \tau_{E}^{L}$, the solid squares have Co-only beam injection, and the open squares have $\tau_{E}<1.5 \tau_{E}^{L}$. 
Fig. 6. The measured global energy confinement time. $\tau_{E}$, plotted against Eq. (3). (A) The $(\mathrm{X})$ points were neither assisted by preceding carbon blooms nor Lithium pellet conditioning, the solid squares were aided by Lithium pellet conditioning, and the open squares were within three (3) shots of Helium discharge cleaning, and the solid squares were within thirty (30) shots of boronization.

Fig. 7. The measured global energy confinement time, $\tau_{E}$, plotted against the central electron density, $\mathrm{n}_{e}(\mathrm{o})$, for $\mathrm{B}=4.8-5.2 \mathrm{~T}, \mathrm{C}_{N B}=0.4-0.7$, and $\beta_{\|} / \beta_{\perp}=1.16-1.28$. The $(\mathrm{X})$ points have $\mathrm{H}_{\alpha}=1.5-2.0 \times 10^{14}$ and the open squares have $\mathrm{H}_{\alpha}=3.0-5.9 \times 10^{14}$.

Fig. 8. The measure global energy confinement time, $\tau_{E}$, plotted as a function of $\beta_{\|} / \beta_{\perp}$ for $\mathrm{B}=4.8-5.2 \mathrm{~T}, \mathrm{C}_{N B}=0.4 \rightarrow 0.7, \mathrm{H}_{\alpha}=6 \rightarrow \tau \times 10^{14}$, and $\mathrm{n}_{e}(\mathrm{o})=5-7 \times 10^{14} \mathrm{~cm}^{-3}$. (A) The data grouped into 10 bins and (B) All of the data points.

Fig. 9. The measured global energy confinement time, $\tau_{E}$, plotted as a function of $\mathrm{C}_{N B}=$ $\mathrm{P}_{C_{0}} / \mathrm{P}_{B}$ for $\mathrm{n}_{e}(0)=5.6 \times 10^{13} \mathrm{~cm}^{-3} . \beta_{\|} / \beta_{\perp}=1.16-1.28$, and $\mathrm{H}_{\alpha}=1.5-3.5 \times 10^{14}$ The data (136 data points) was grouped into 8 bins and the error bars are standard deviations of the mean.

Fig. 10. The measured global energy confinement time, $\tau_{E}$, plotted against the $\mathrm{H}_{\alpha}, \beta_{\|} / \beta_{\perp}$, and $\mathrm{n}_{e}(\mathrm{o})$ dependences of Eq. (3) for $\mathrm{C}_{N B}=0.5 \rightarrow 0.75$. The $(\mathrm{X})$ points have $\mathrm{B}=4.8$ $5.2 \mathrm{~T}$ and the open squares have $\mathrm{B}=3.9 \mathrm{~T}$. (A) The data was grouped into 20 intervals and (B) all the data.

Fig. 11. The ratio of the measured global energy confinement time to Eq. (3) plotted as a function of the deuterium recycling light, $\mathrm{H}_{\alpha}$. 
Fig. 12. The ratio of the measured global energy-confinement time to Eq. (3) plotted as a function of the confinement enhancement over L-mode.

Fig. 13. Time evolution of the relevant parameters for two plasmas from Table 1.

(A) pressure anisotropy, $\beta_{\|} / \beta_{\perp}$ : solid line is 52969 , dashed line is 52990 ,

(B) deuterium recycling light, $\mathrm{H}_{\alpha}$ : solid line is 52969 , dashed line is 52990 .

(C) total energy content, E: solid line is 52969 , dashed line is 52990 ,

(D) central electron density, $\mathrm{n}_{e}(0)$ in units of $10^{13} \mathrm{~cm}^{-3}$ : solid line is 52969 , dashed line is 52990 ,

(E) confinement time for 52990: solid line is measurement. dashed line is Eq. (3),

(F) confinement time for 52969: solid line is measurement, dashed line is Eq. (3).

Fig. 14. Time evolution of the relevant parameters for two plasmas from Table 1.

(A) pressure anisotropy, $\beta_{\|} / \beta_{\perp}: 49928$ (solid) and 52950 (dashed),

(B) deuterium recycling light, $\mathrm{H}_{\alpha}: 49928$ (solid) and 52950 (dashed),

(C) total energy content, E: 49928 (solid) and 52950 (dashed),

(D) central electron density, $\mathrm{n}_{e}(\mathrm{o})$ in units of $10^{13} \mathrm{~cm}^{-3}: 49928$ (solid) and 52950 (dashed),

(E) confinement time, $\tau_{E}$, for 49928: measured (solid) and Eq. (3) (dashed),

(F) confinement time, $\tau_{E}$, for 52950: measured (solid) and Eq. (3) (dashed).

Fig. 15. Time evolution of the relevant parameters for the two plasmas from Table 2 .
(A) pressure anisotrcpy, $\beta_{\|} / \beta_{\perp}: 51354$ (solid) and 55848 (dashed),
(B) deuterium recycling light, $\mathrm{H}_{\alpha}: 51354$ (solid) and 55848 (dashed),
(C) Total energy content, E: 51354 (solid) and 55848 (dashed),
(D) central electron density, $\mathrm{n}_{e}(\mathrm{o})$ in units of $10^{13} \mathrm{~cm}^{-3} .51354$ (solid) and 5.5848 (dashed),
(E) confinement time, $\tau_{E}$, for 51354: measured (solid) and Eq. (3) (dashed), 
(F) confinement time, $\tau_{E}$, for 55848: $\operatorname{measured}^{-}$(solid), and Eq. (3) (dashed).

Fig. 16. Measured global energy confinement time plotted as a function of Eq. (3) for the time evolution data from the plasmas of Tables 1 and 2. The $(\mathrm{X})$ points are from Table 1, the $(+)$ points are 55848 and the open squares are 51354.

Fig. 17. Time evolution of the relevant parameters for two plasmas from Table 3.

(A) pressure anisotropy, $\beta_{\|} / \beta_{\perp}: 47725$ (solid) and 51254 (dashed),

(B) deuterium recycling light, $\mathrm{H}_{\alpha}: 47725$ (solid) and 51254 (dashed),

(C) total energy content. E: 47725 (solid) and 51254 (dashed).

(D) central electron density, $\mathrm{n}_{e}(\mathrm{o})$ in units of $10^{14} \mathrm{~cm}^{-3}: 47725$ (solid) and 51254 (dashed),

(E) confinement time, $\tau_{E}$, for 47725: measured (solid) and Eq. (3) (dashed),

(F) confinement time, $\tau_{E}$, for 51254: measured (solid) and Eq. (3) (dashed).

Fig. 18. Time evolution of the relevant parameters for two plasmas from Table 3 . (A) pressure anisotropy, $\beta_{\|} / \beta_{\perp}$, (B) deuterium recycling light, $\mathrm{H}_{\alpha},(\mathrm{C})$ total energy content, $E$, and central density $\mathrm{n}_{e}(\mathrm{o})$ in units of $10^{20} \mathrm{~cm}^{-3}$ for 51383 (solid) and 55683 (dashed). The confinement time for (E) 51383 and (F) 5.5683 compares measured values (solid) with Eq. (3) (dashed).

Fig. 19. The measured confinement time, $\tau_{E}$, plotted against Eq. (3) for the time evolution of the plasmas in Table 3 ( $\mathrm{X}$ points) and Table 4 (open square points).

Fig. 20. Time evolution of the relevant parameters for two of the plasmas in Table 4 . (A) the pressure anisotropy, $\beta_{\|} / \beta_{\perp}$, (B) the deuterium recycling light, $\mathrm{H}_{\alpha},(\mathrm{C})$ the total energy content, E, (D) the central electron density $\mathrm{n}_{e}(\mathrm{o})$ in units of $10^{14} \mathrm{~cm}^{-3}$ with 55850 (solid) and 51267 (dashed). The measured energy confinement (solid) is compared to Eq. (3) 
for (E) 51267 and (F) 55850 .

Fig. 21. Time evolution of the relevant plasma parameters from 48041 (Table 4). (A) pressure anisotropy, $\beta_{\|} / \beta_{\perp}$, (B) deuterium recycling light, $\mathrm{H}_{\alpha},(\mathrm{C})$ central electron density, $\mathrm{n}_{e}(\mathrm{o})$ in units of $10^{13} \mathrm{~cm}^{-3}$, and (D) the confinement time: measured (solid) and Eq. (3) (dashed).

Fig. 22. Measured global energy confinement time plotted against the deuterium recycling light for a bloom and MHD free plasma (open squares), plasma experiencing a bloom $(\mathrm{X})$ points. and plasma experiencing strong $\mathrm{MHD}(+)$ points.

Fig. 23. The time evolution of the plasma parameters for the plasma aided by a preceding bloom (Table 5). The plasma parameters are (A) the pressure anisotropy, $\beta_{\|} / \beta_{\perp},(\mathrm{B})$ the deuterium recycling light, $\mathrm{H}_{\alpha},(\mathrm{C})$ the central density $\mathrm{n}_{e}(\mathrm{O})$ in units of $10^{13} \mathrm{~cm}^{-3}$, and (D) the energy confinement time [measured (solid) and from Eq. (3) (dashed)].

Fig. 24. The time evolution of the plasma parameters for a plasma aided by a preceding lithium pellet and its comparison shot (Table 5). The plasma parameters are: (A) the pressure anisotropy, $\beta_{\|} / \beta_{\perp}$, (B) the deuterium recycling light, $\mathrm{H}_{\alpha},(\mathrm{C})$ the total energy content, E. and (D) the central density, $\mathrm{n}_{e}(\mathrm{o})$ in units of $10^{13} \mathrm{~cm}^{-3}$, where for each case 53782 (solid) is the comparison plasma and 53843 (dashed) is the plasma aided by a lithium pellet. The energy confinement is shown in (E) for 53843 and $(F)$ for 53782 where the measured value (solid) is compared to Eq. (3) (dashed).

Fig. 25. The time evolution of the plasma parameters aided by a preceding lithium pellet and its comparison plasma (Table 5). The plasma parameters are: (A) the pressure anisotropy, $\beta_{\|} / \beta_{\perp}$, (B) the deuterium recycling light, $H_{\alpha}$, (C) the total energy content, 
E. and (D) the central density, $n_{e}(0)$, in units of $10^{13} \mathrm{~cm}^{-3}$. The solid line represents 55605 , the comparison plasma and the dashed line represents 55632 , the plasma aided by the lithium pellet. The energy confinement time, $\tau_{E}$, is plotted in (E) for 55605 and (F) for 55632 where the solid line is the measured value and the dashed line is Eq. (3).

Fig. 26. Time evolution of $(A)$ neutral beam power, $P_{B},(B)$ total energy content. E. (C) density peakedness, $F_{n}$, and (D) the neutron emission, $S_{n}$ for the $0.8 \mathrm{MA}$ overdrive plasma (47789) (solid) and the comparison plasma (47701) (dashed) of Table 6.

Fig. 27. Time evolution of the plasma parameters for the overdrive plasma (477S9) (solid) and its comparison plasma (47791) (dashed) (Table 6). (A) is the pressure anisotropy, $\beta_{\|} / \beta_{\perp},(\mathrm{B})$ is the deuterium recycling light, $\mathrm{H}_{\alpha},(\mathrm{C})$ is the central electron density, $\mathrm{n}_{\mathrm{e}}(\mathrm{o})$ in units of $10^{13} \mathrm{~cm}^{-3}$. The energy confinement is plotted in D with the 47789 measured value (dashed-dot line) and 47791 (solid line) and Eq. (3) for 47789 (long dashed line) and for 47791 (short dashed line).

Fig. 28. Measured global energy confinement time, $\tau_{E}$, plotted against Eq. (3) for the 1.4 MA overdrive plasmas $(\mathrm{X})$ points and their comparison plasmas (solid squares).

Fig. 29. The time evolution of the plasma parameters for the deuterium pellet target plasma (55053) and its supershot comparison (53848) from Table $T$. The plasma parameters are (A) the pressure anisotropy, $\beta_{\|} / \beta_{\perp}$, the deuterium recycling light, $H_{\alpha},(\mathrm{C})$ the total energy content, $E$, and (D) the central electron density. $\mathrm{n}_{e}(\mathrm{o})$, in units of $10^{13} \mathrm{~cm}^{-3}$. The solid lines are 53848 and the dashed lines are 55053. The confinement times for (E) 53848 and $(F) 55053$ are plotted as solid lines for the measured values and dashed lines for Eq. (3). 
Fig. 30. The transport coefficients as a function of minor radius from the energy balance analysis for the supershots with low $\tau_{E}$ (solid line) and high $\tau_{E}$ (dashed line).

Fig. 31. The electron particle diffusivity plotted against the L-mode energy confinement time, $\tau_{E}^{L}$, at $25 \mathrm{~cm}(\mathrm{X})$ points, $40 \mathrm{~cm}$ (open squares), and $60 \mathrm{~cm}$ (solid circles). The data points have been grouped into 9 intervals and represent 118 analyses of the transport coefficients. The error bars are the standard deviation of the mean for the data in each interval.

Fig. 32. The particle diffusivity at $40 \mathrm{~cm}$ (half-radius) as a function of beam power for plasma currents $I_{p}=1.4-1.7 \mathrm{MA}(\mathrm{X})$ points, $I_{p}=1.0 \mathrm{MA}$ (open squares), and $I_{p}=1.85-2.0$ MA (solid circles). 


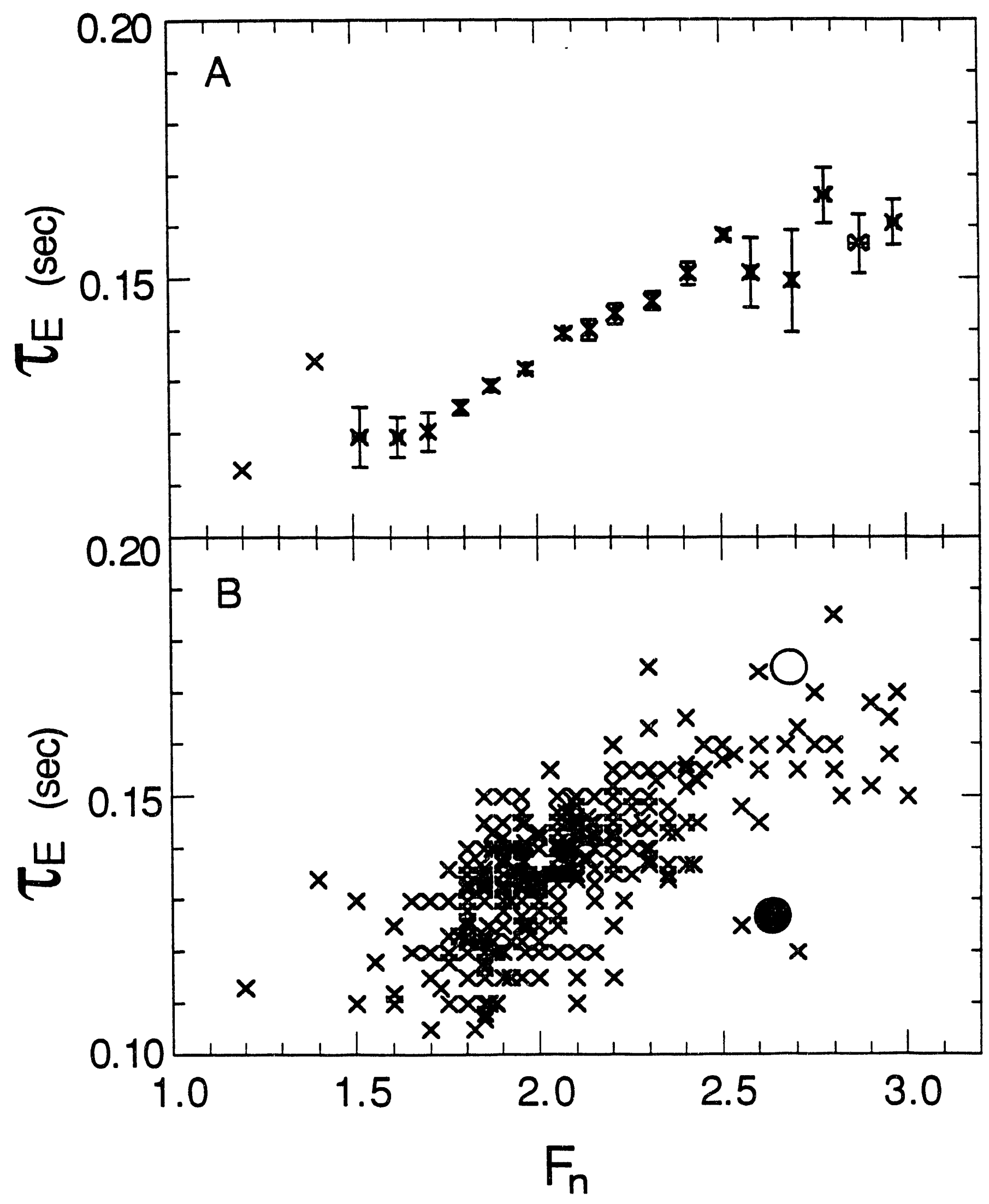

Fig. 1 


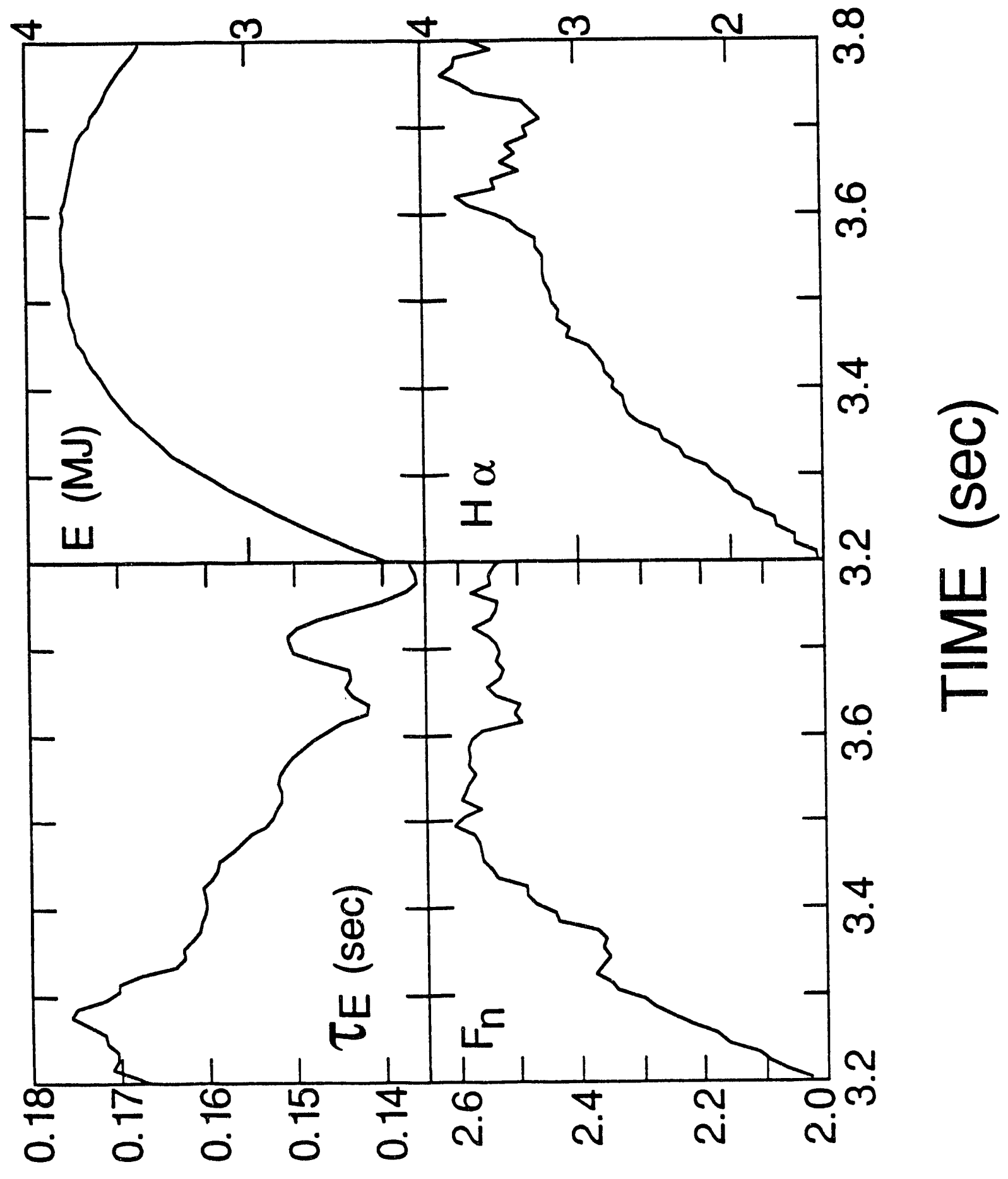

Fig. ? 


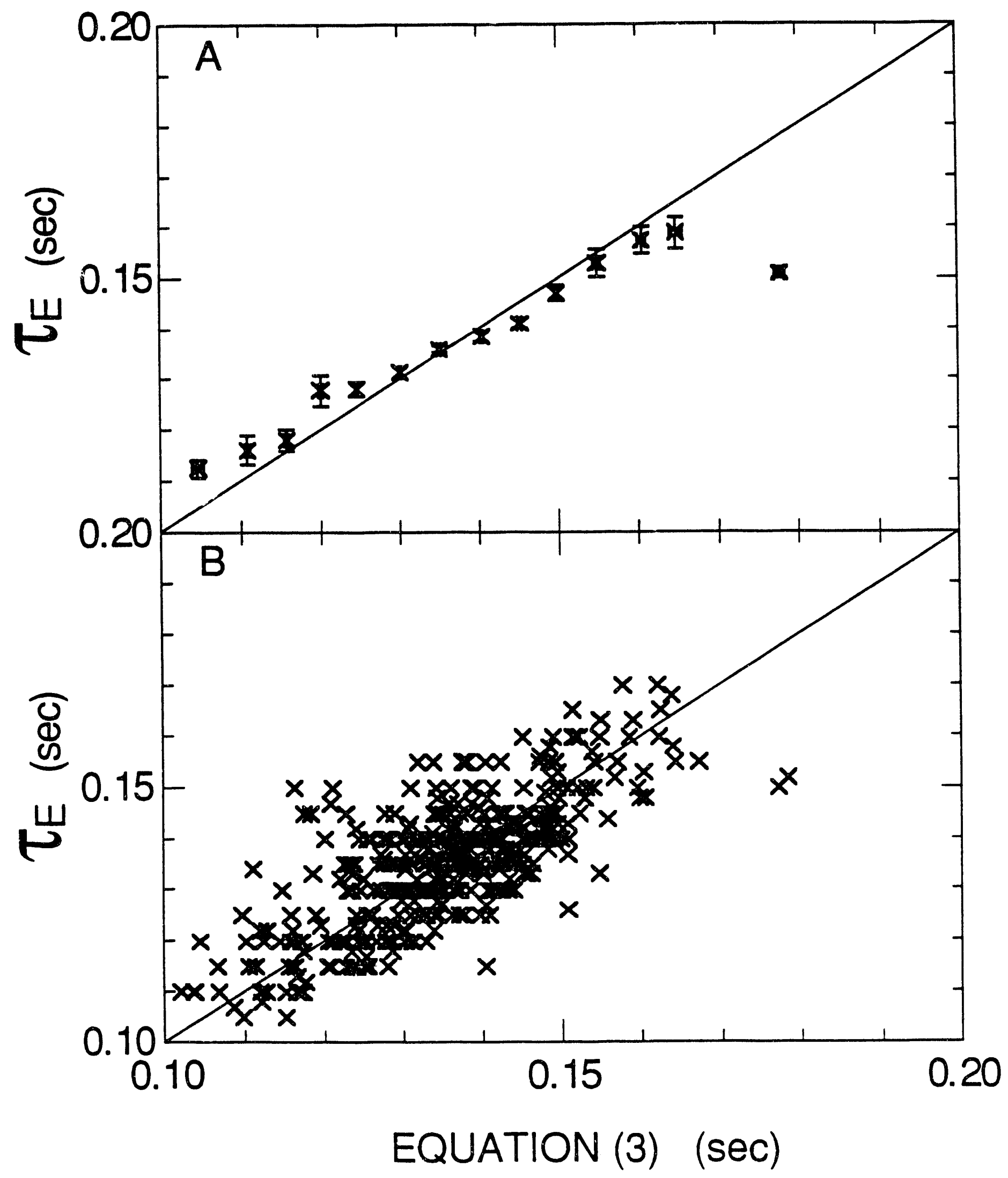

Fig. 3 


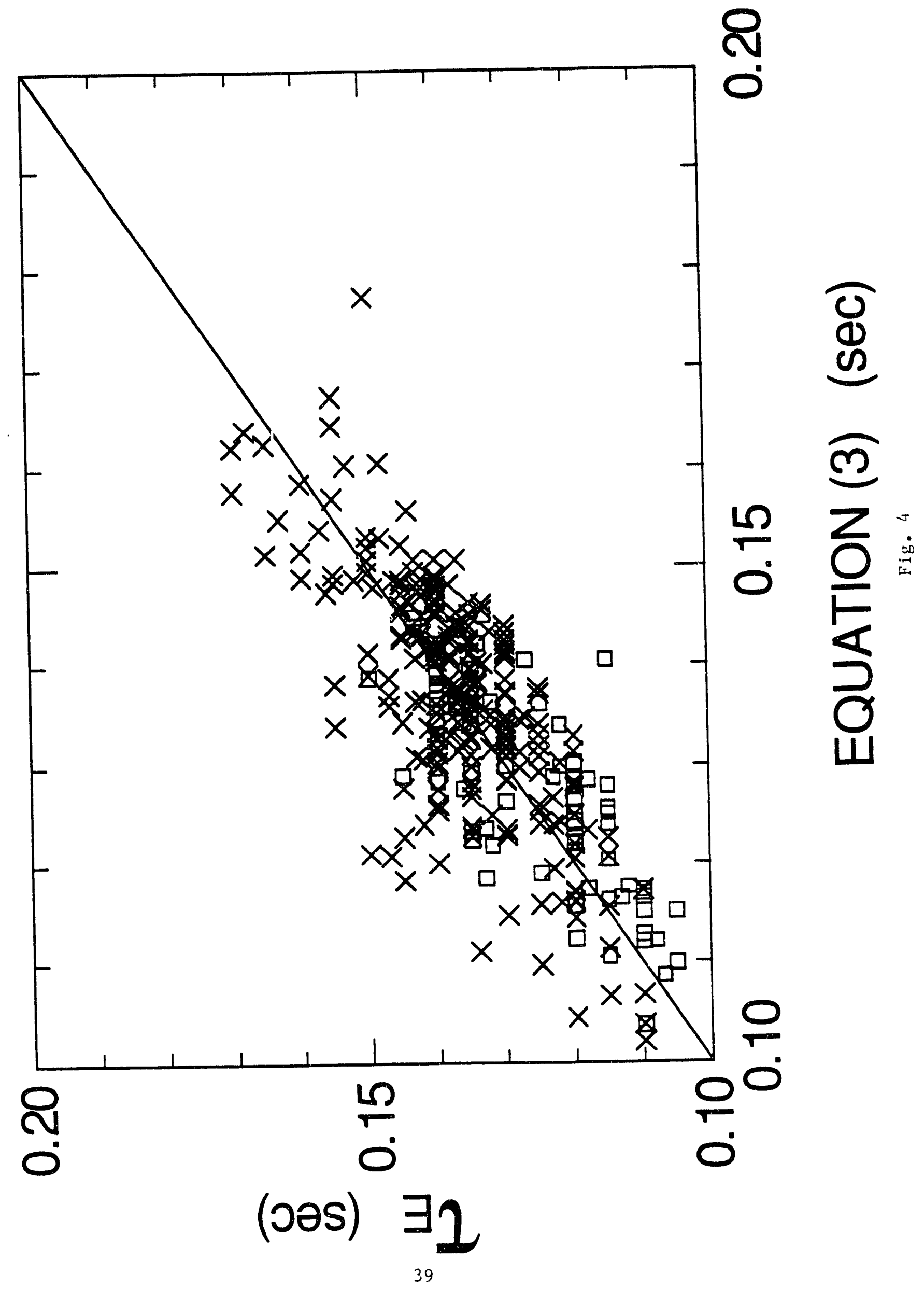




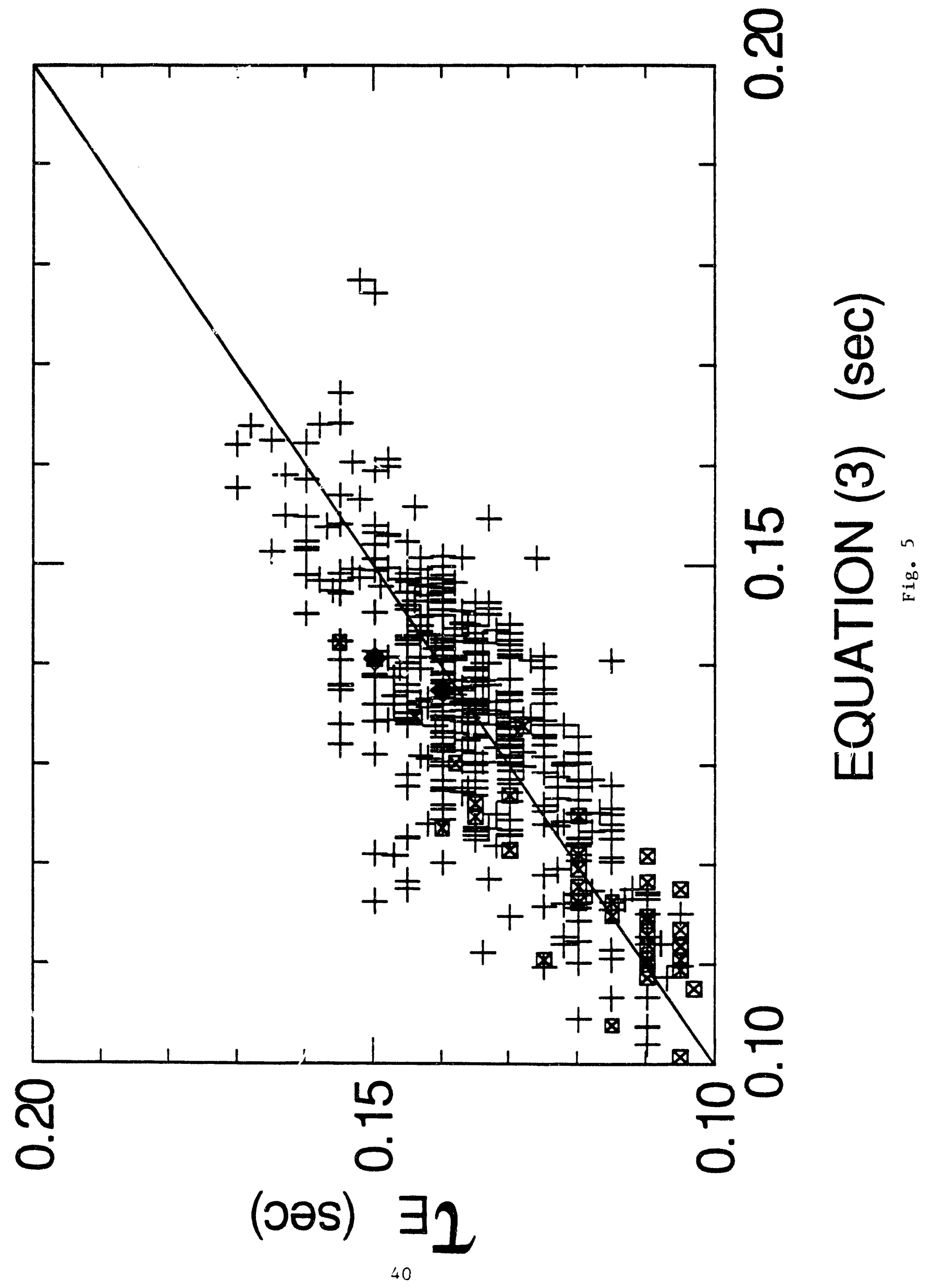




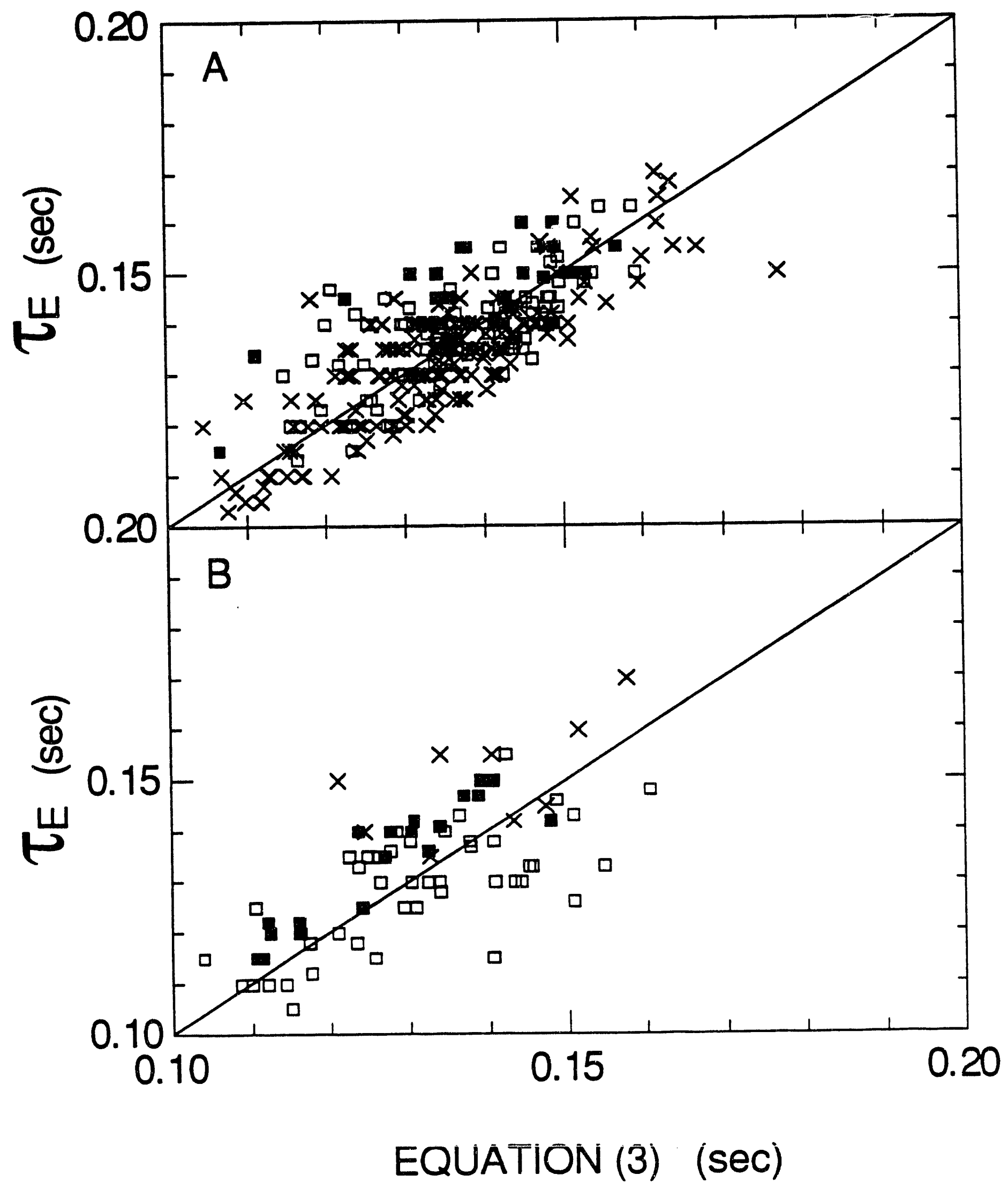

Fig. 6 


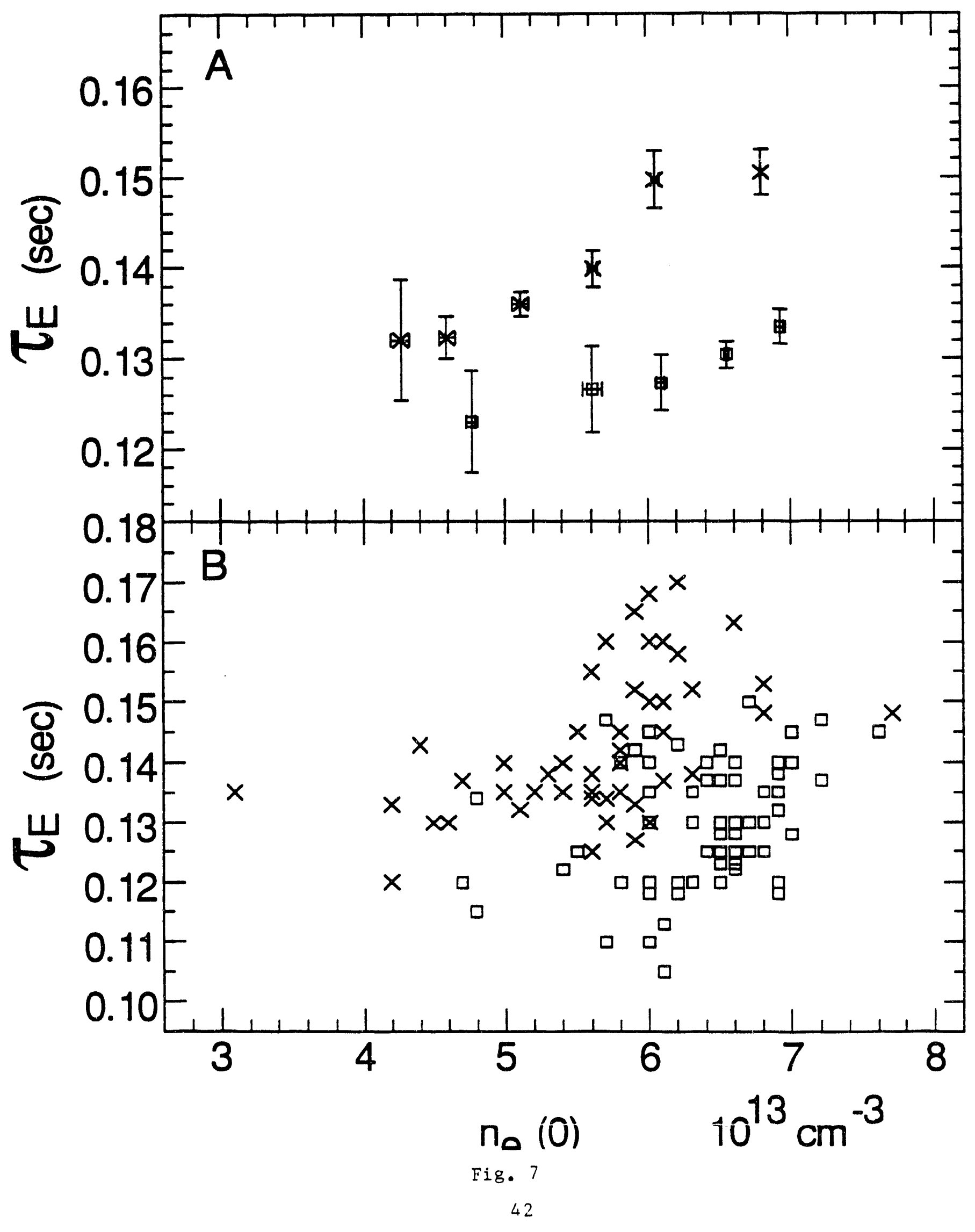




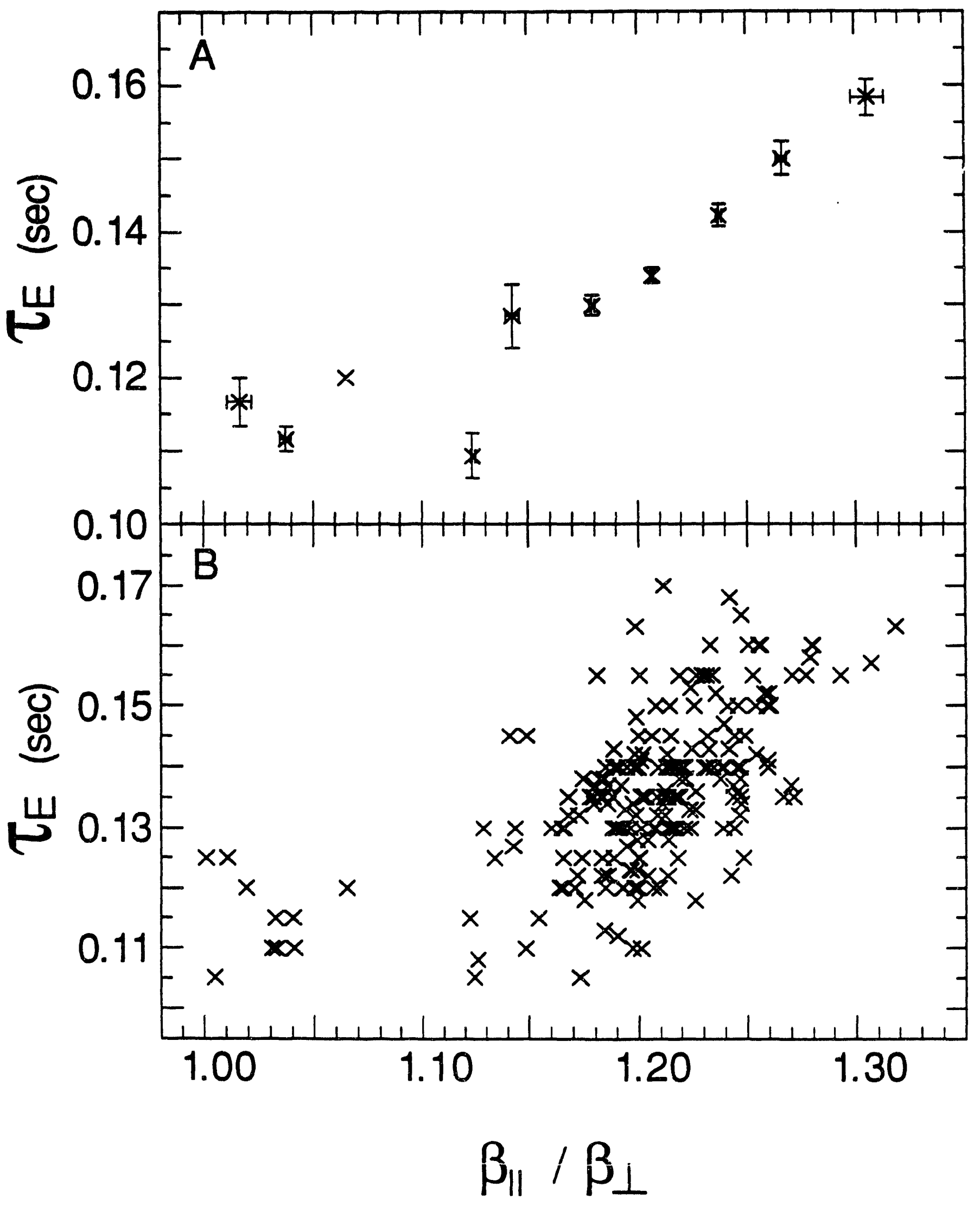

Fig. 8 


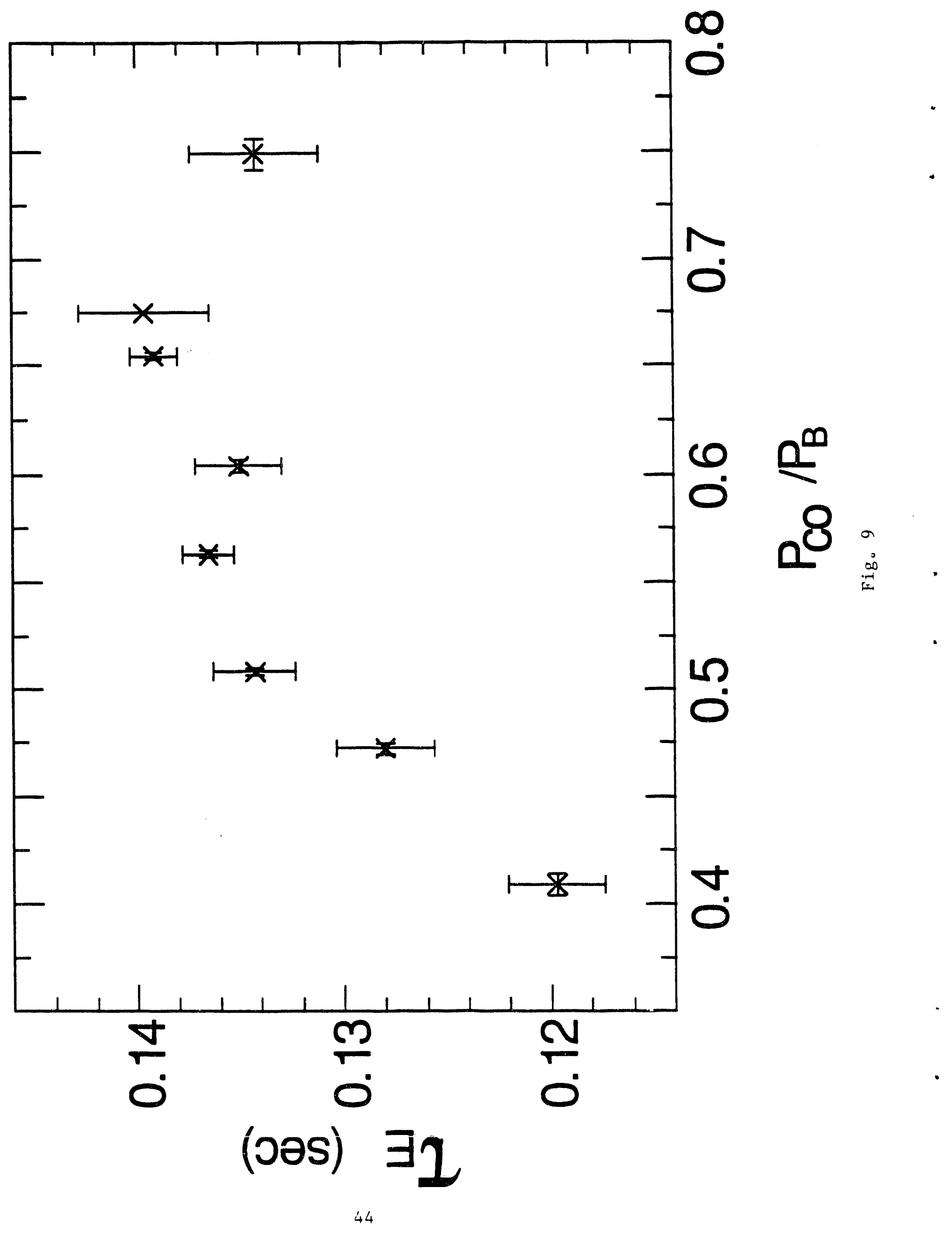




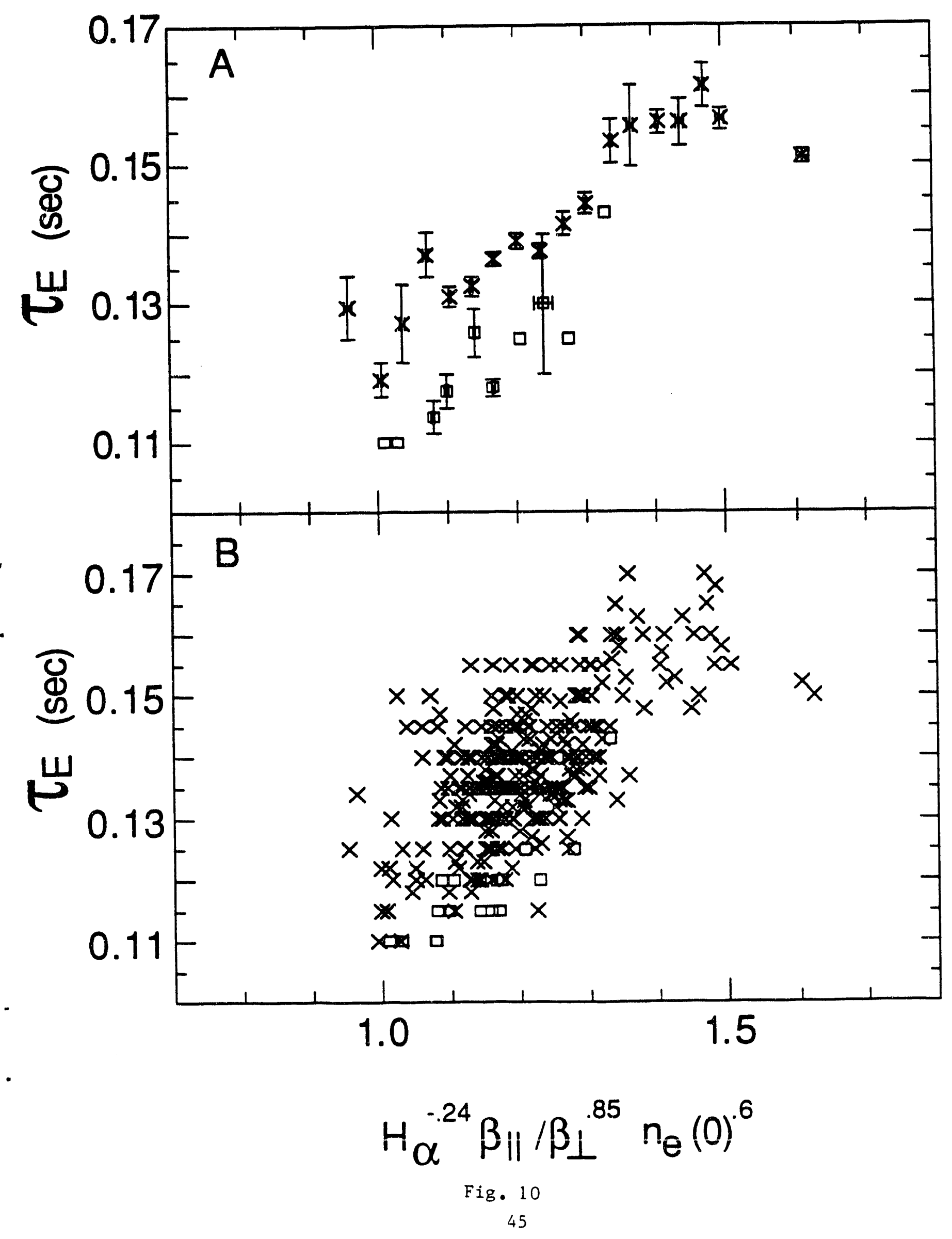




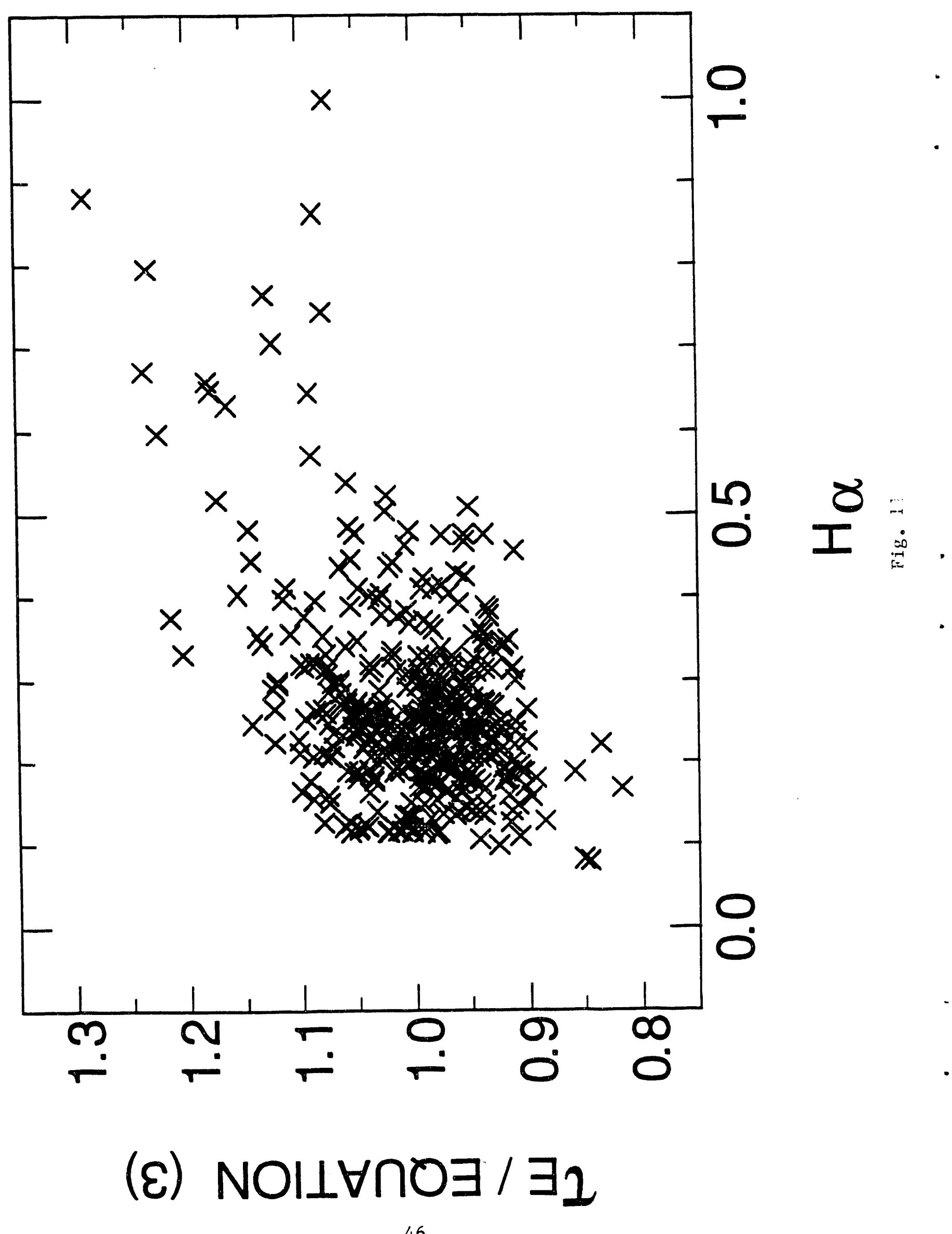




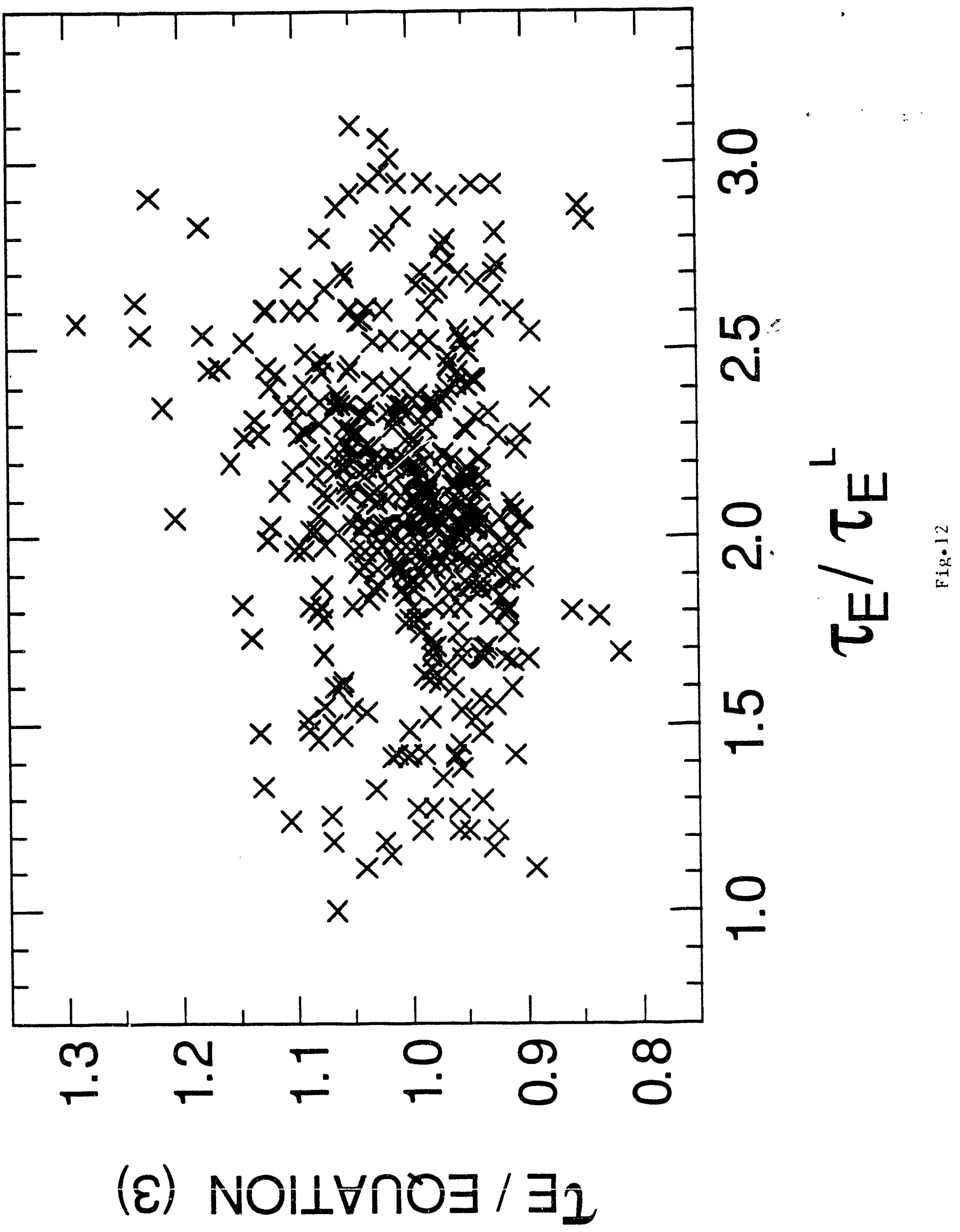




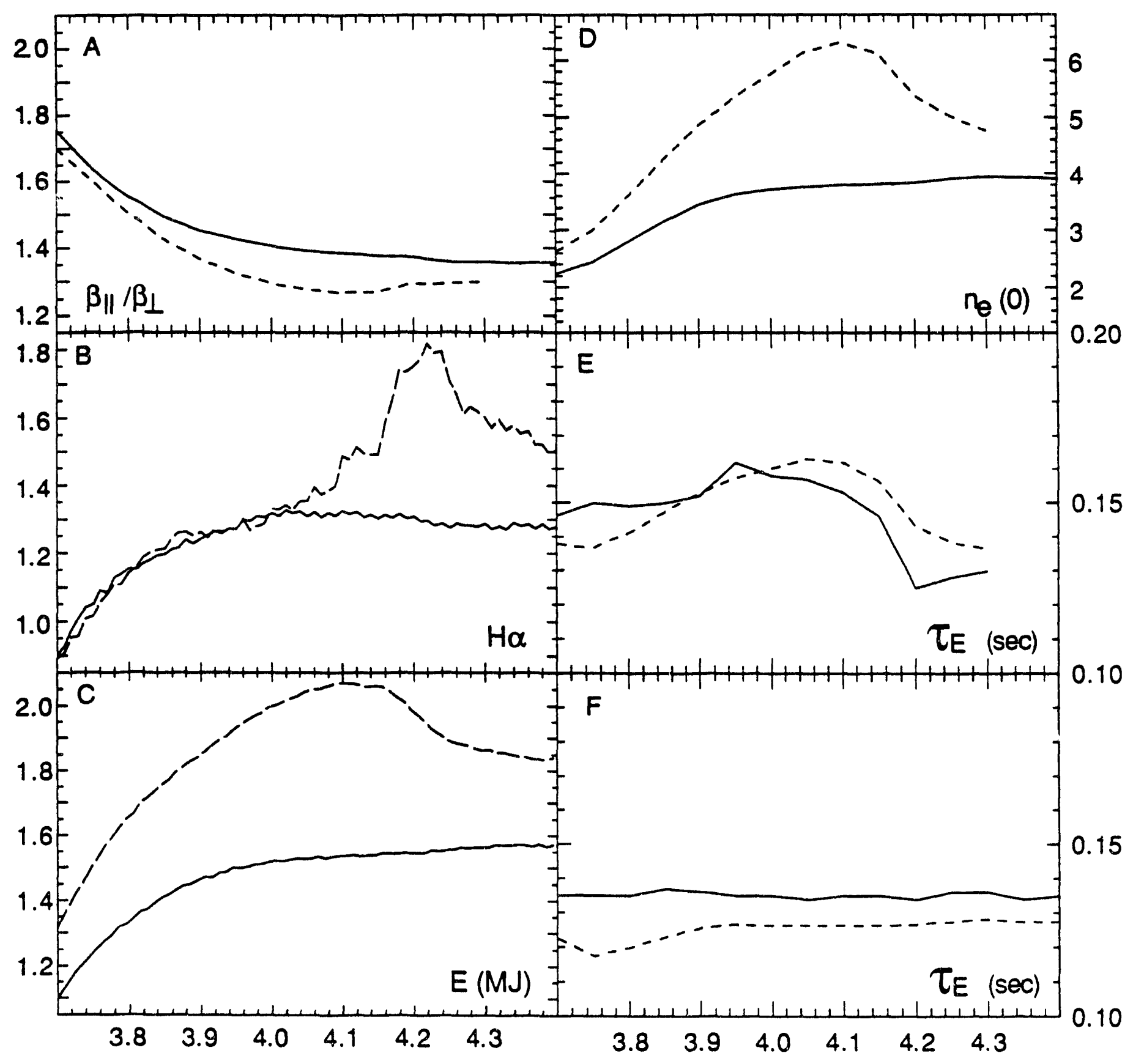

TIME (sec) 


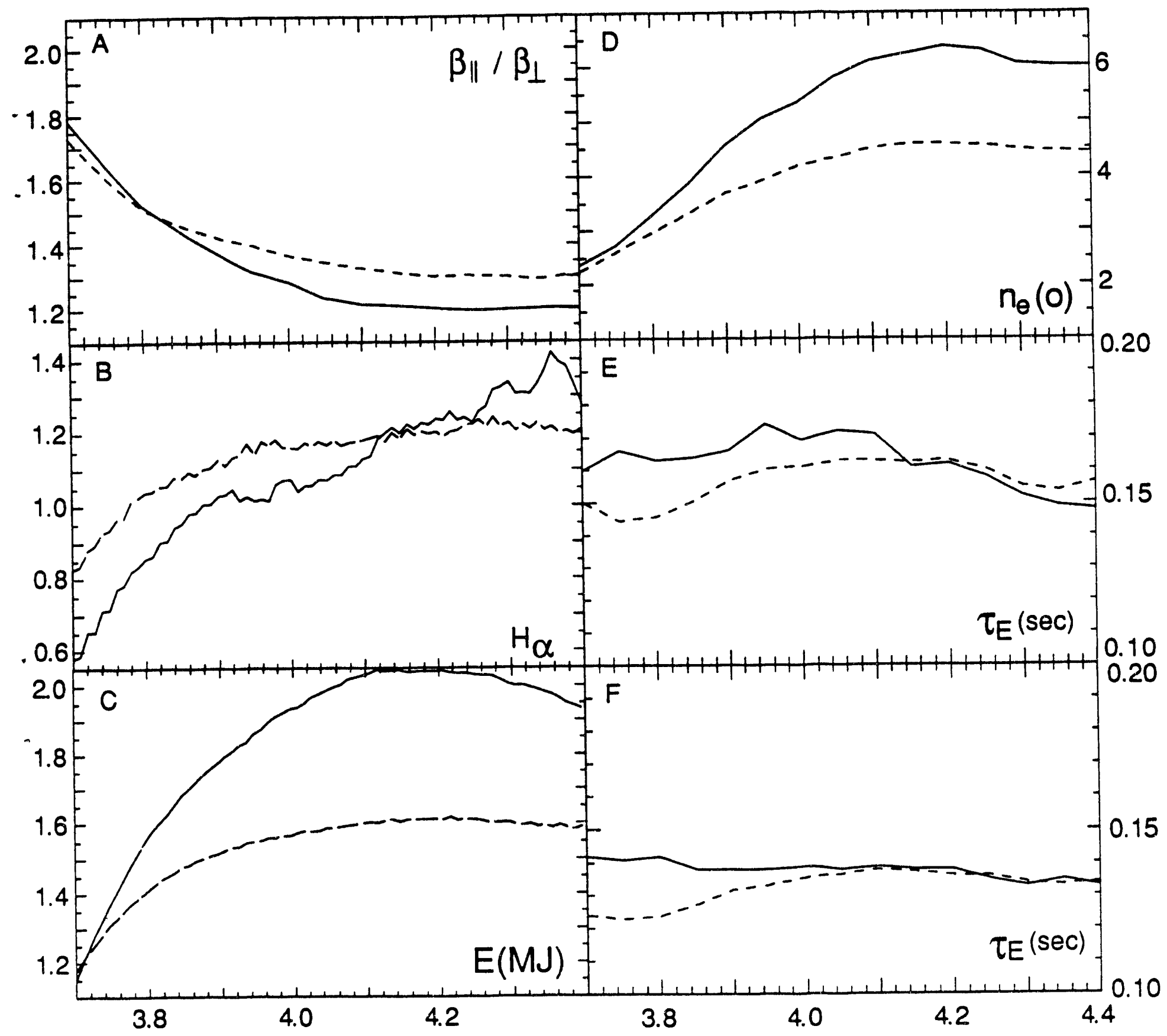

TIME (sec) 


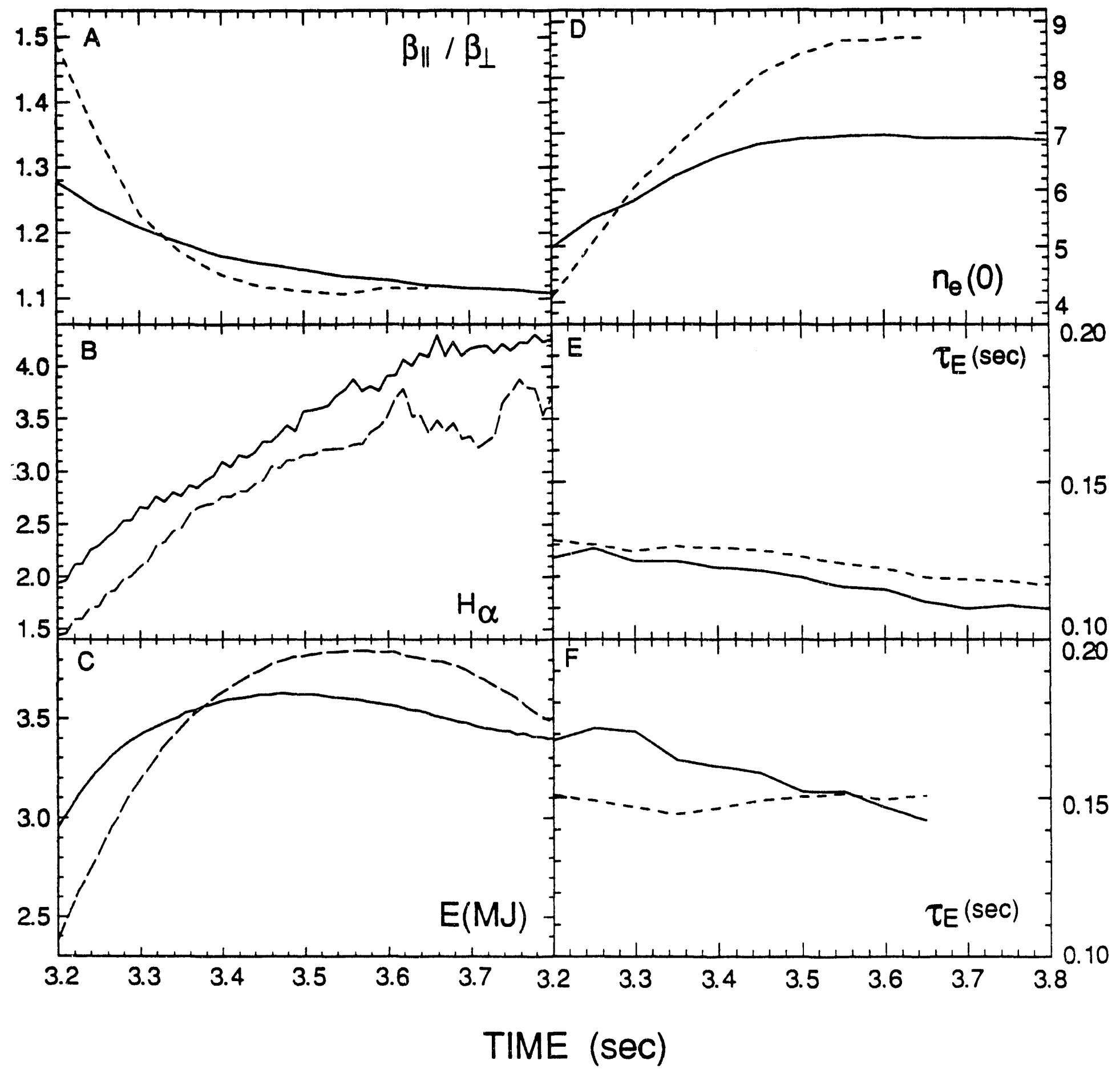

Fig. 15 


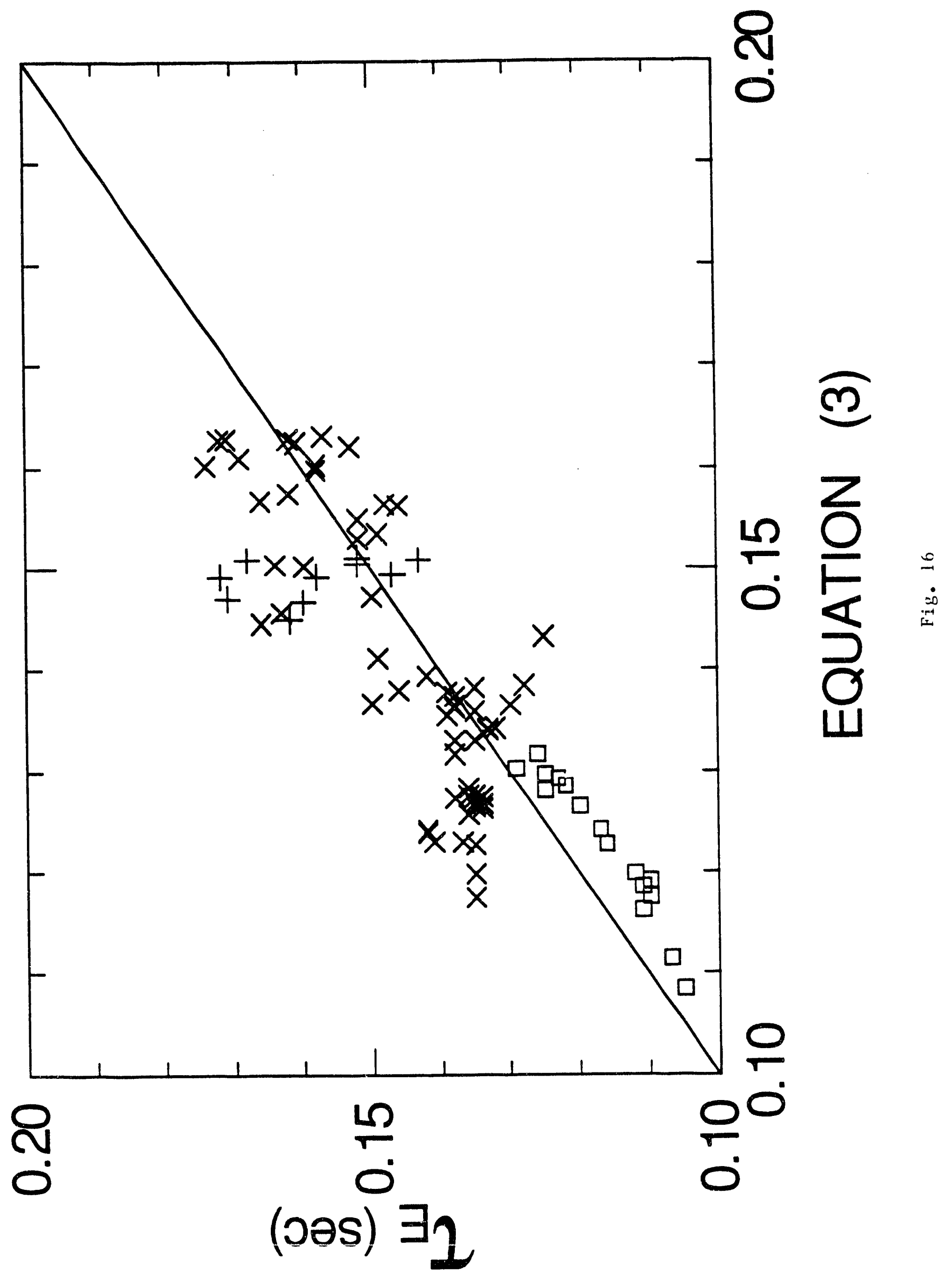




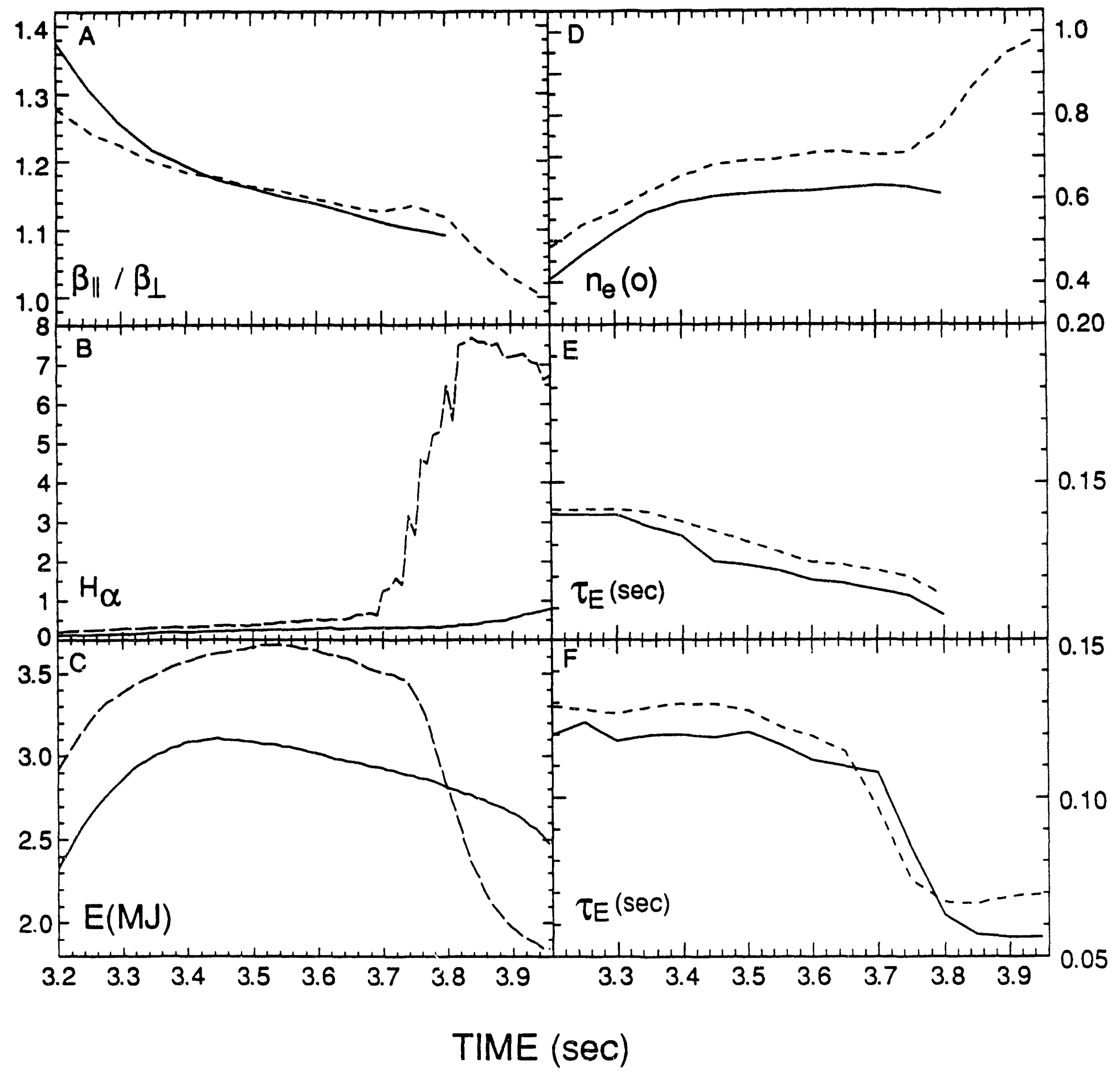

Fig。 17 


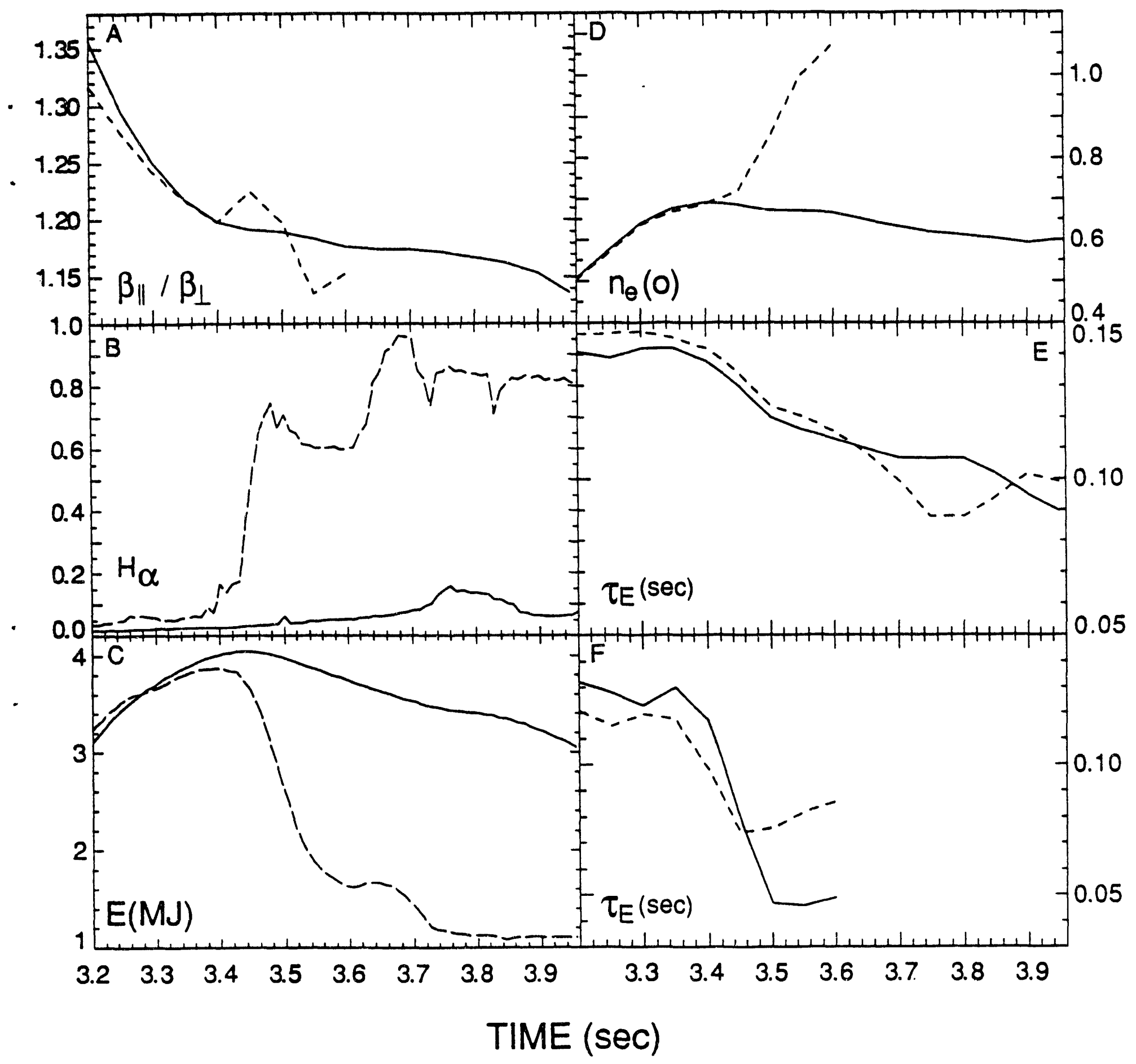




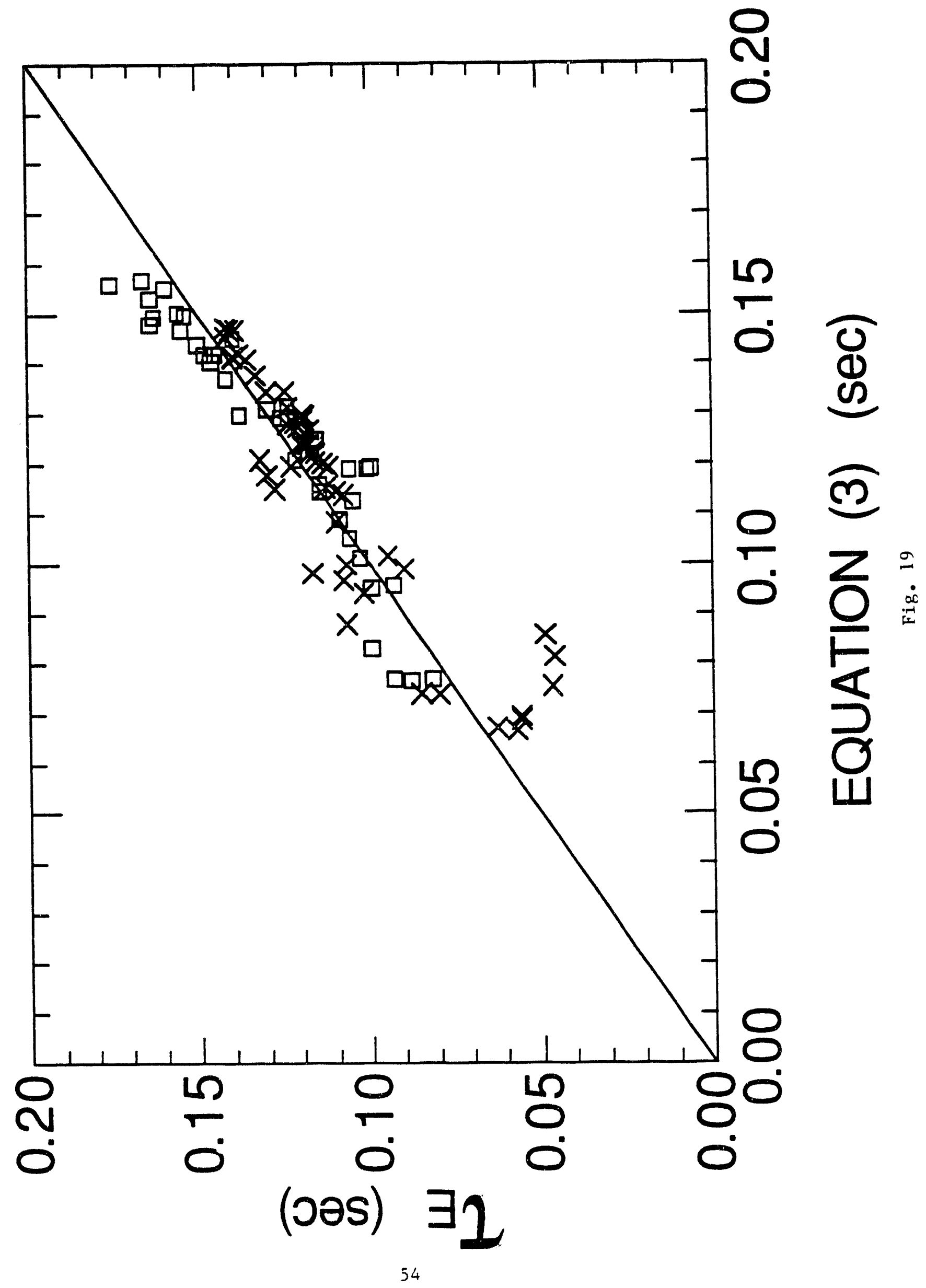




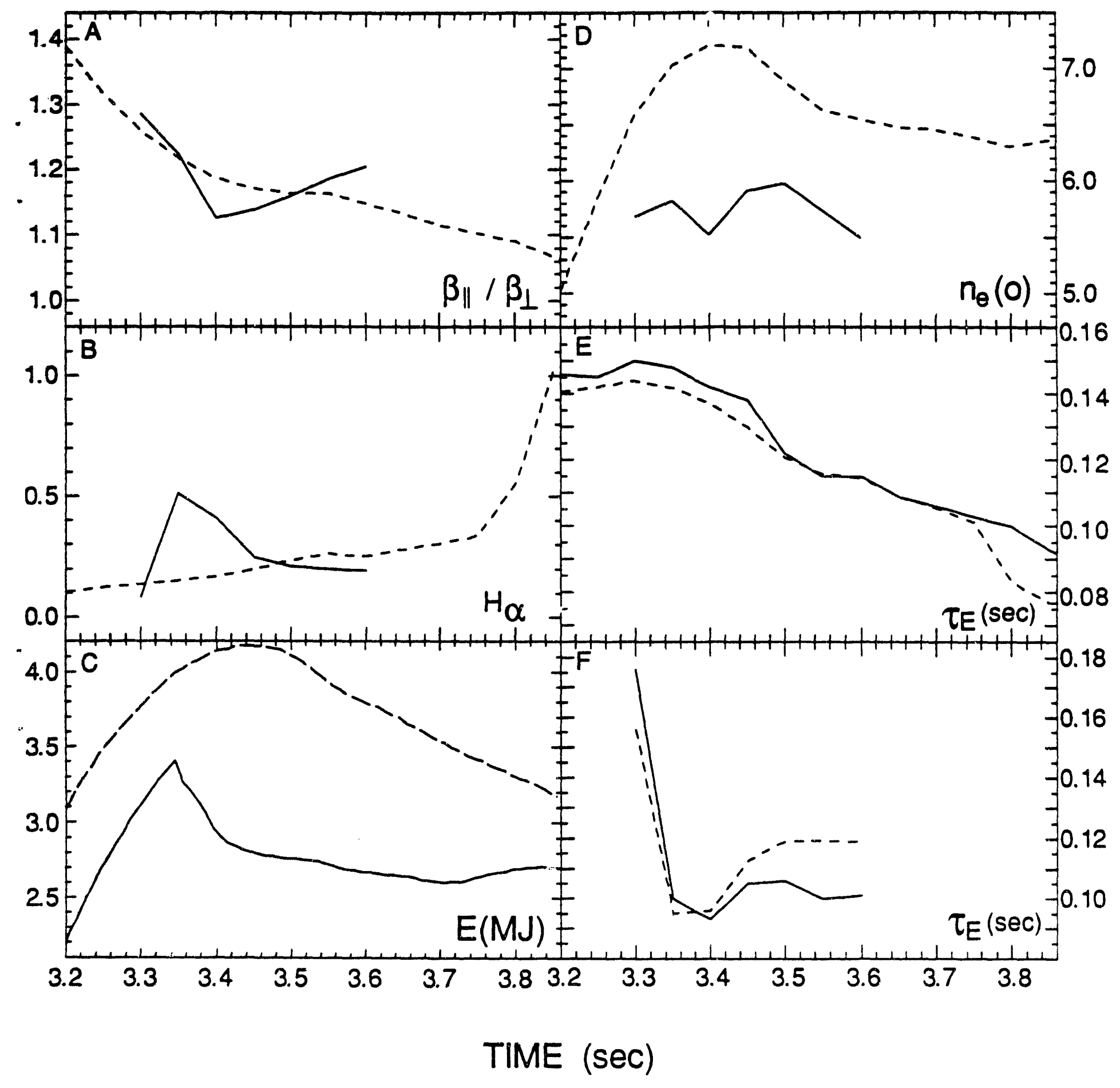

Fig. 20 


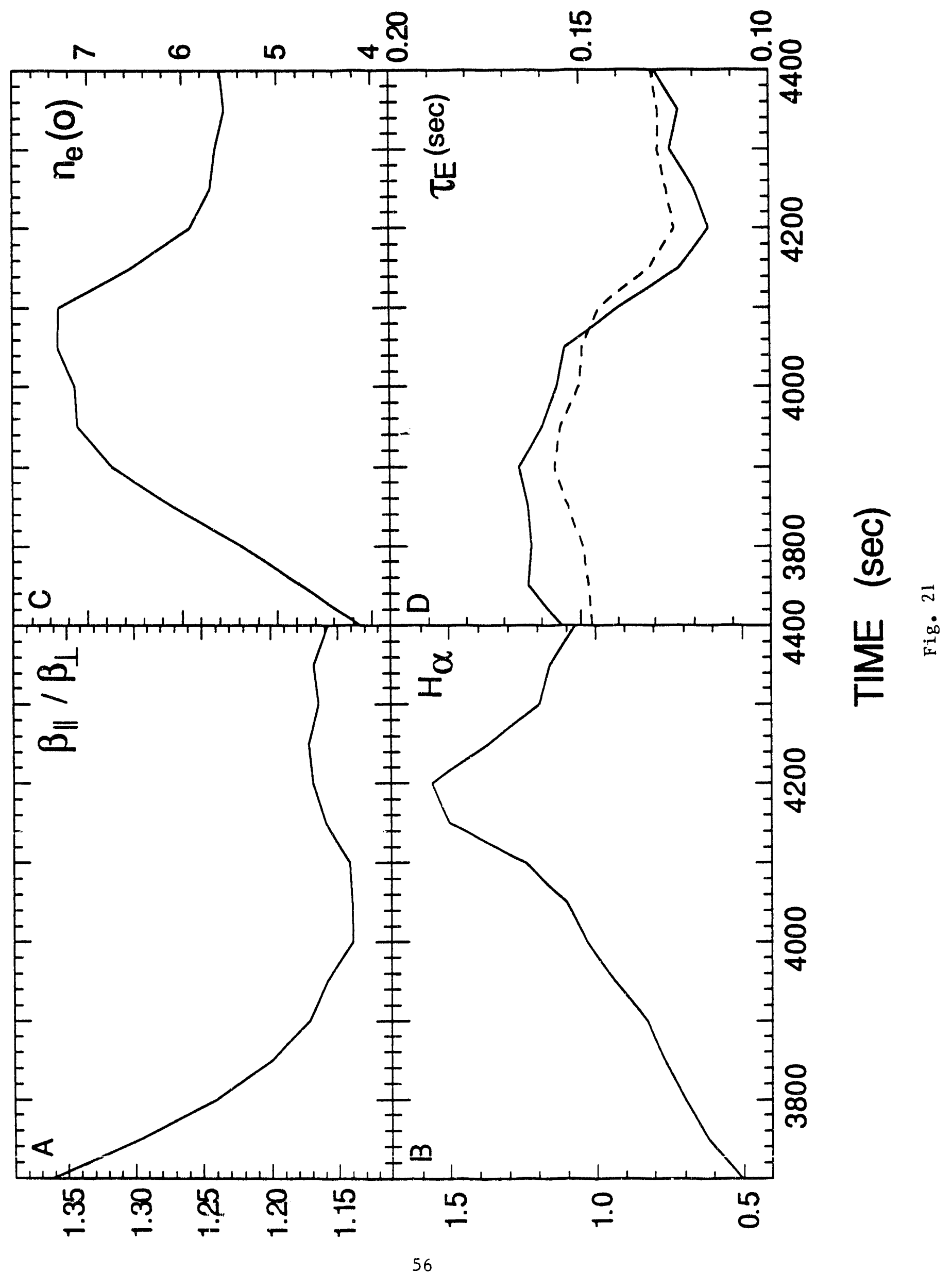




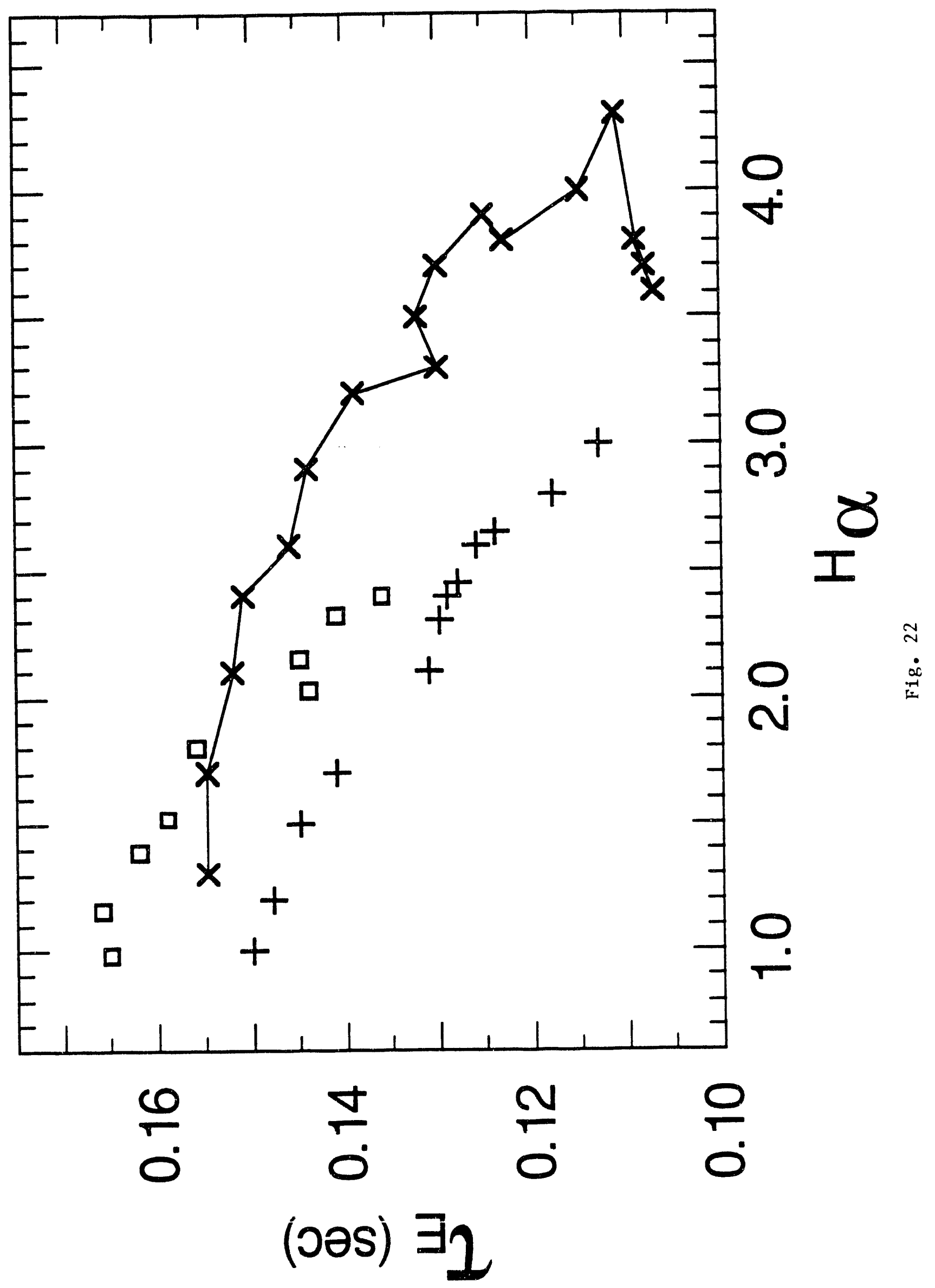




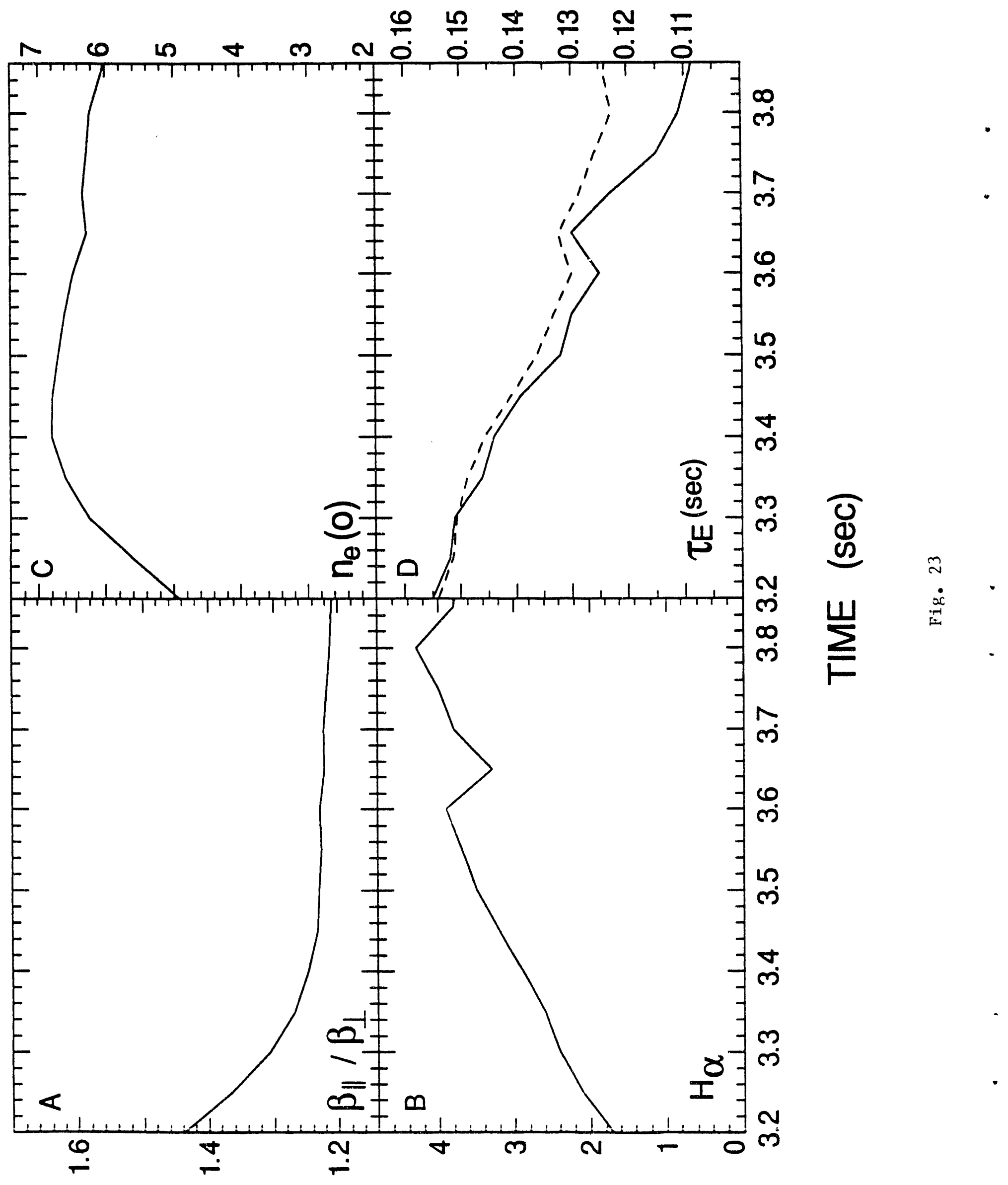




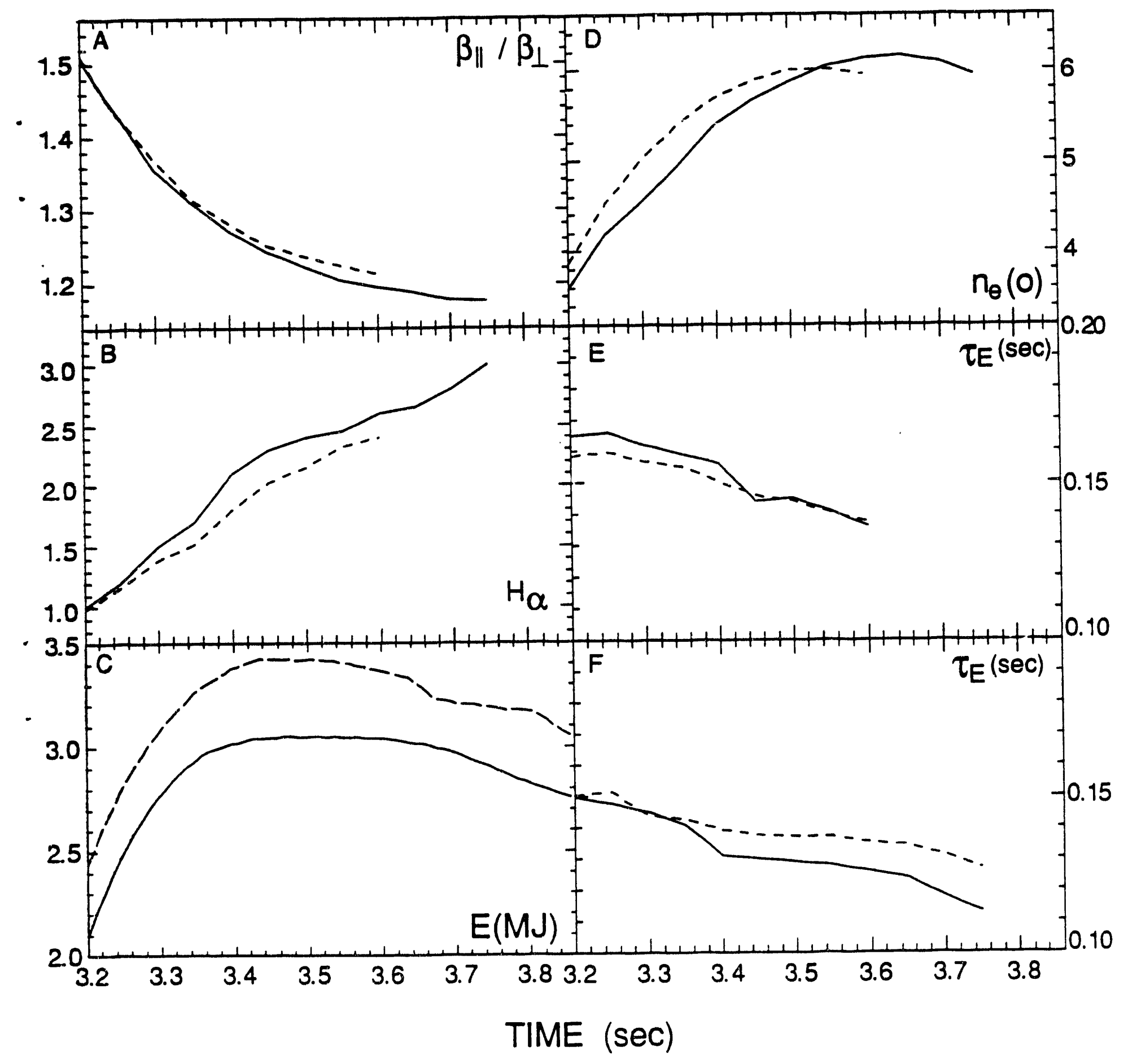

Fig. 24 


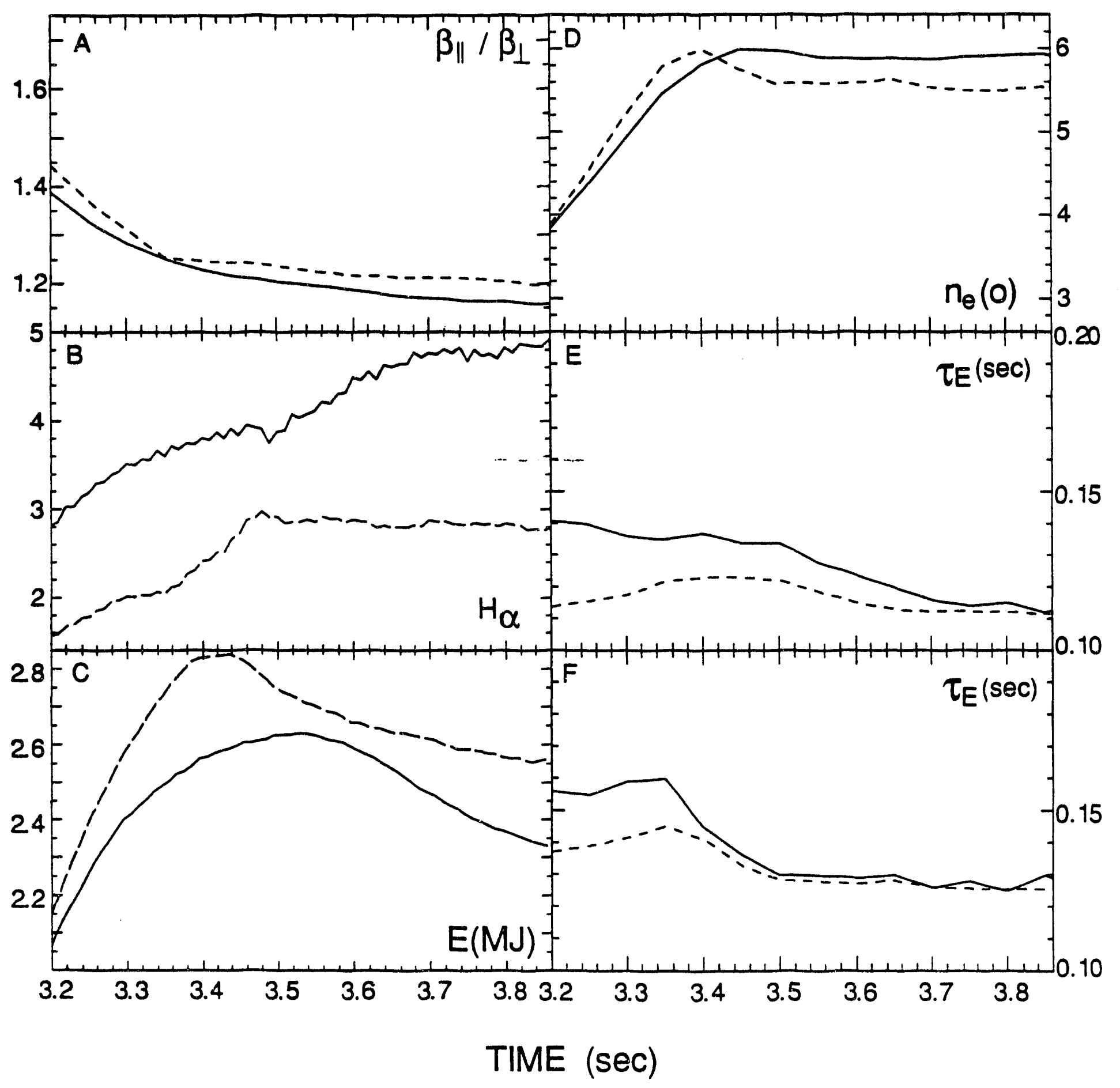

Fig. 25 


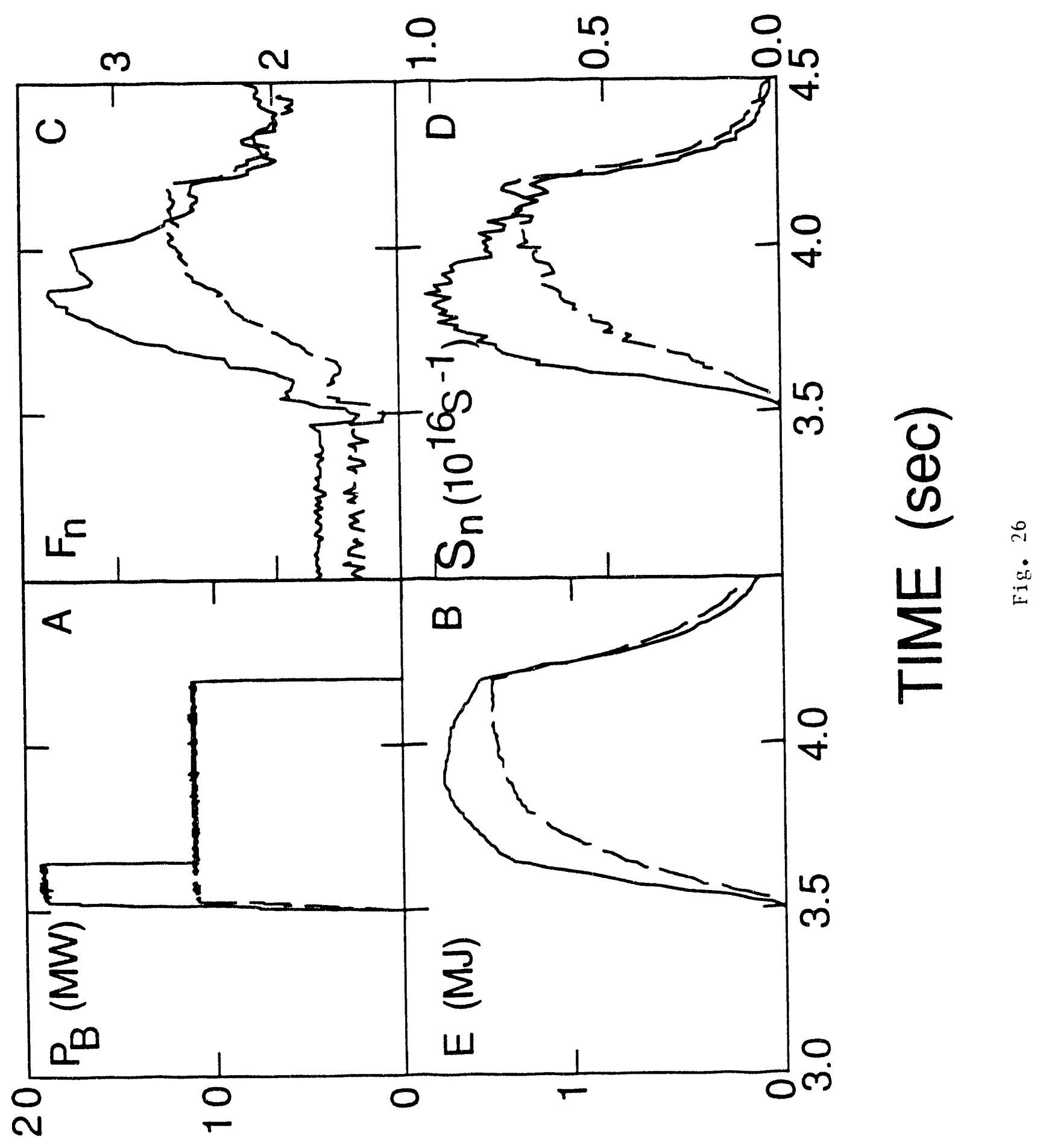




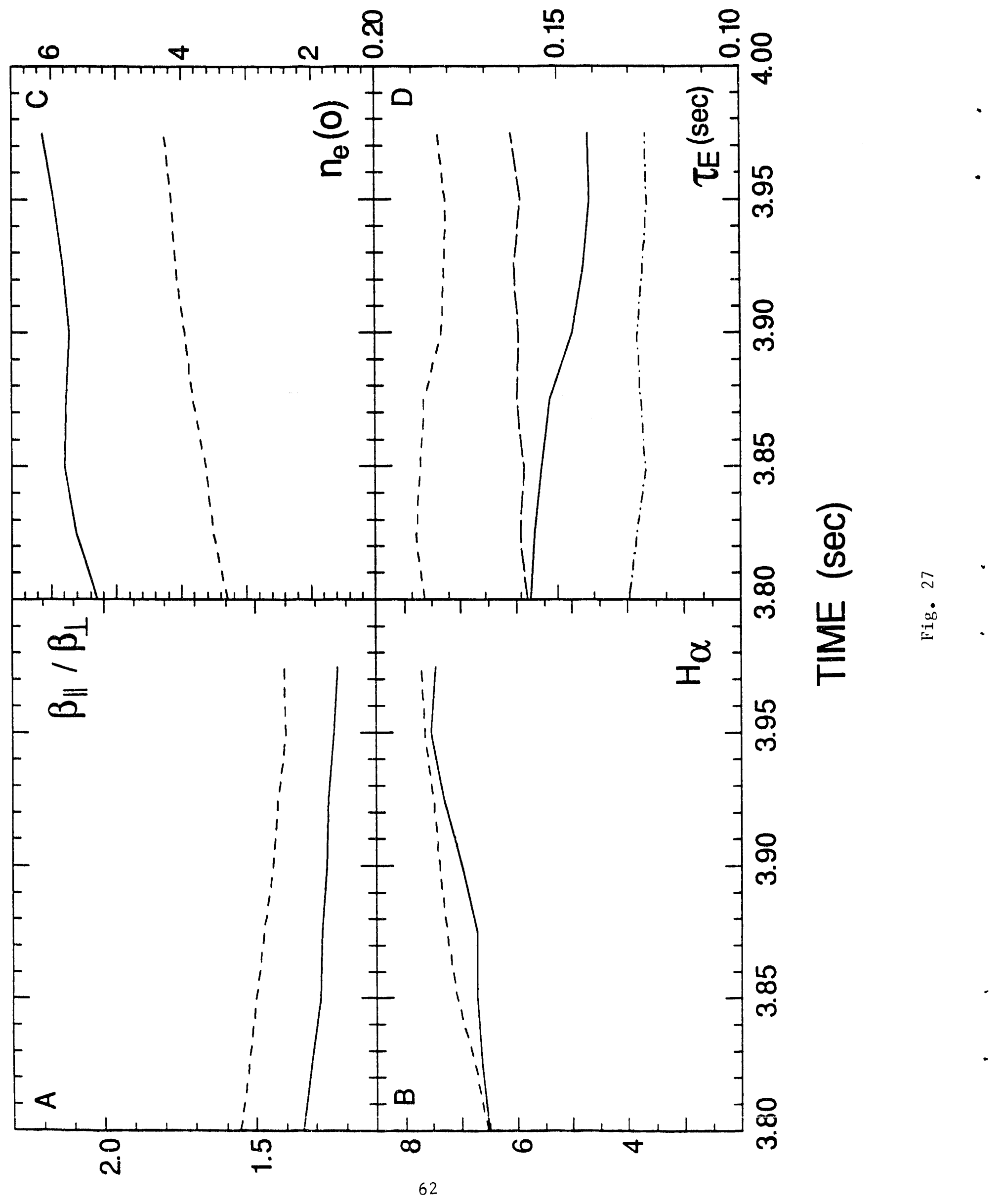




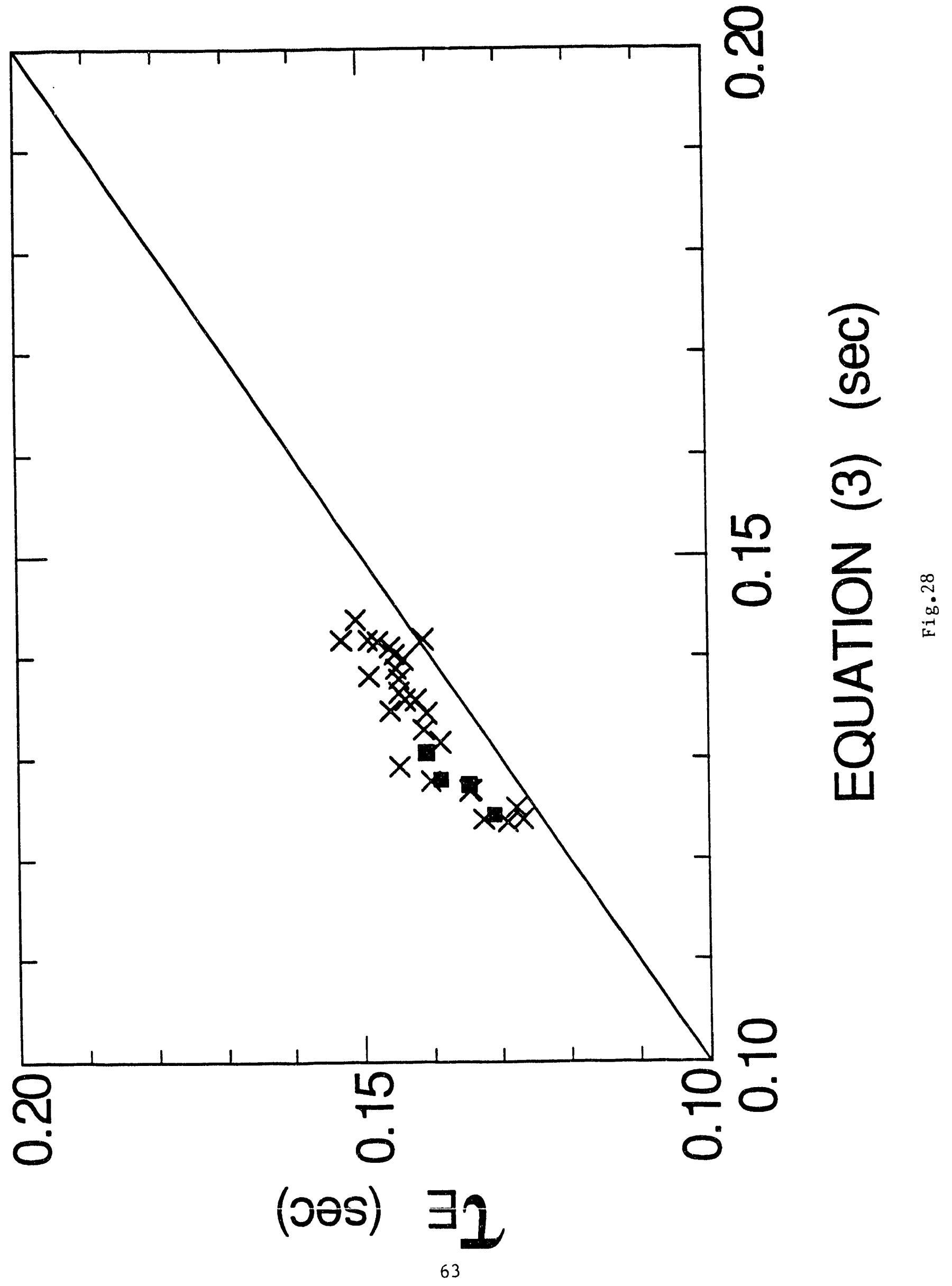




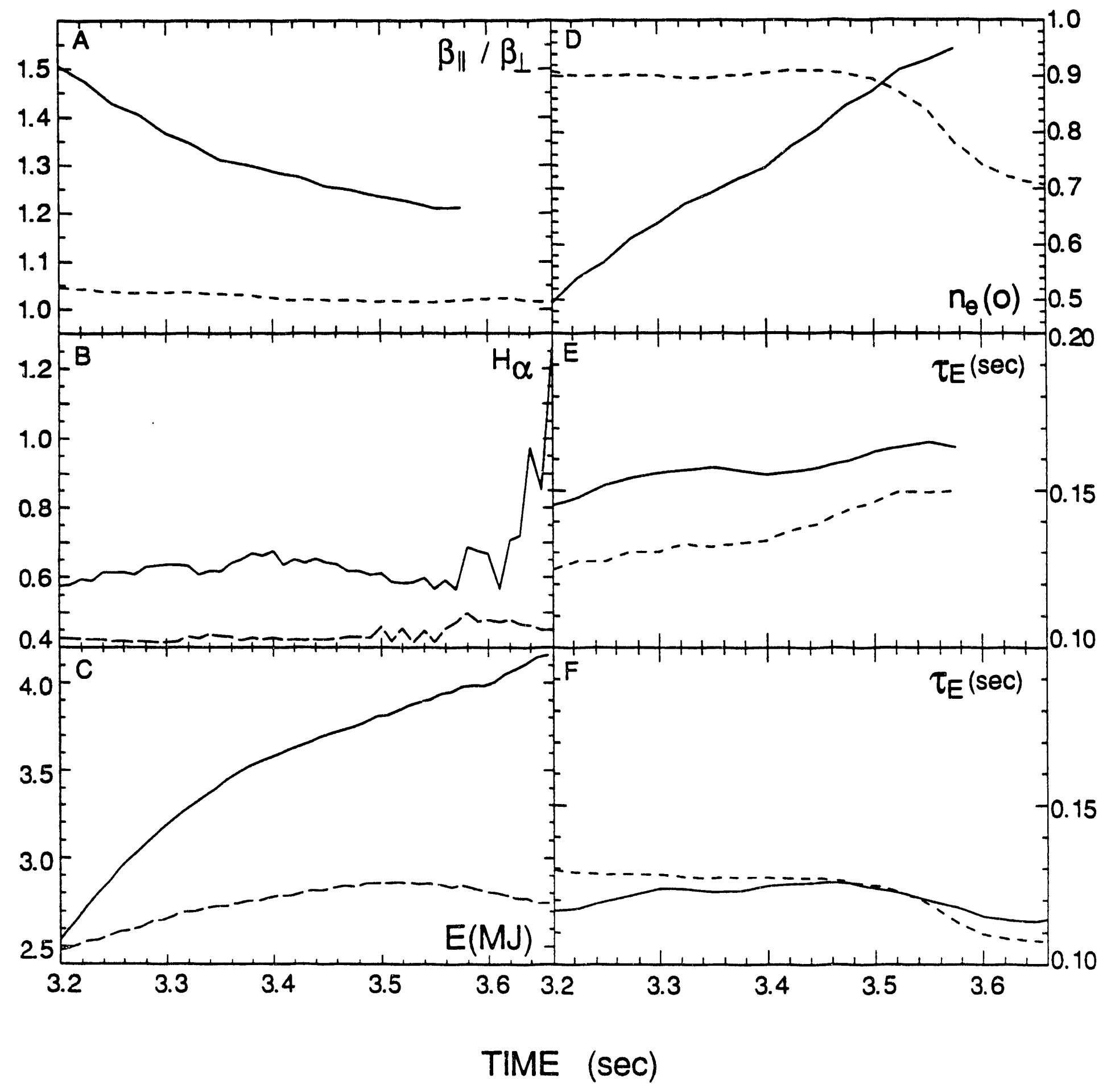




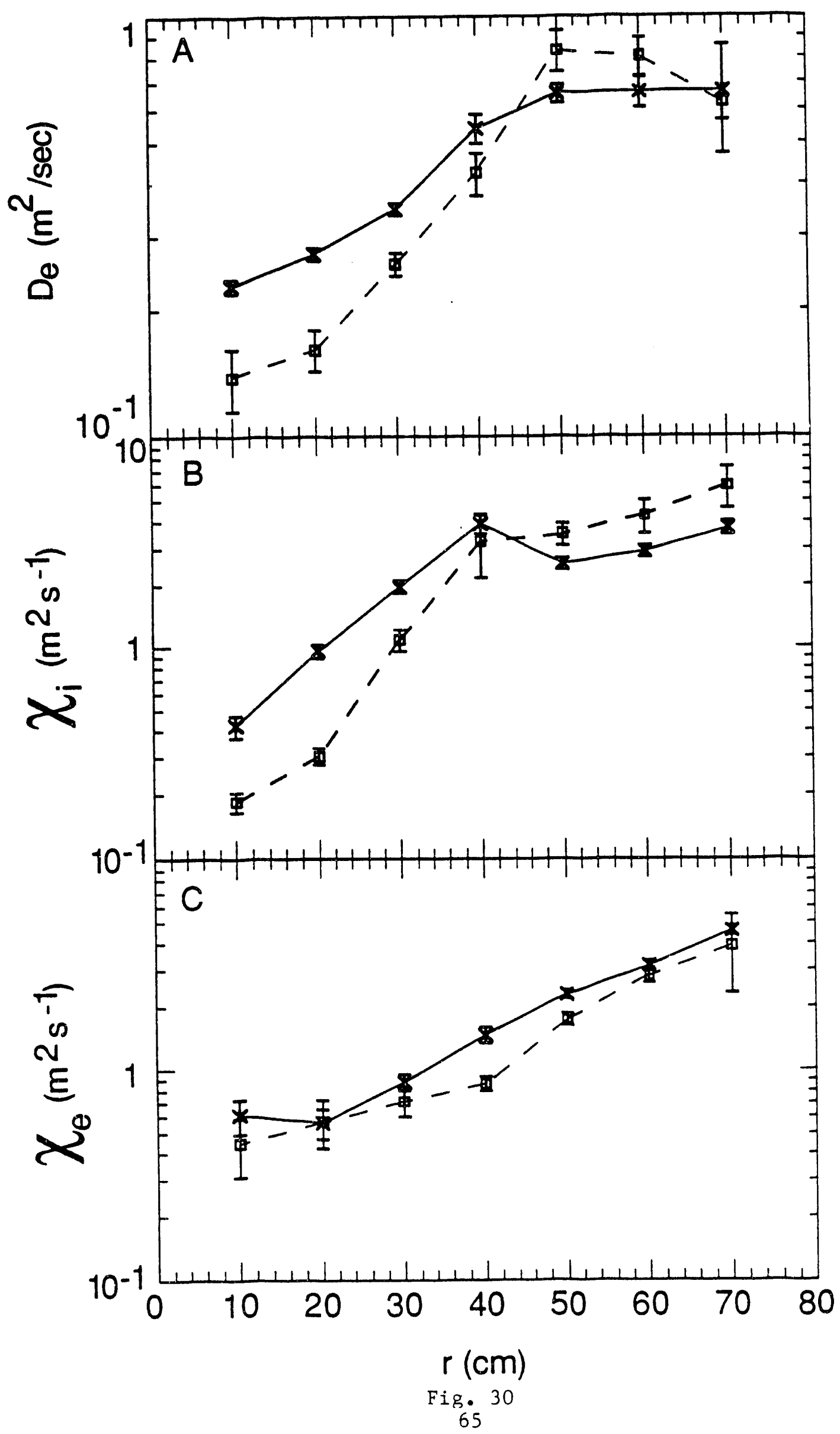




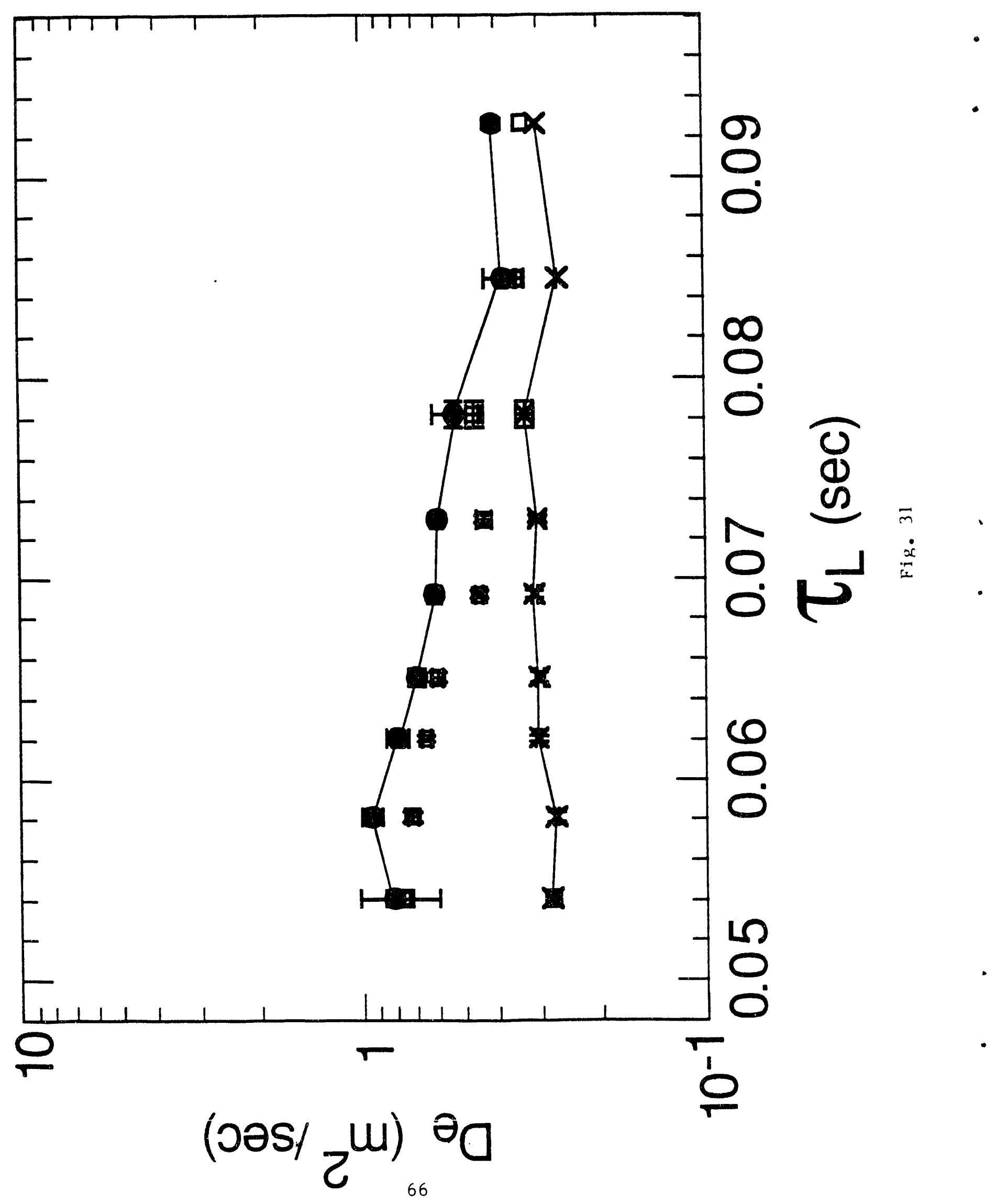




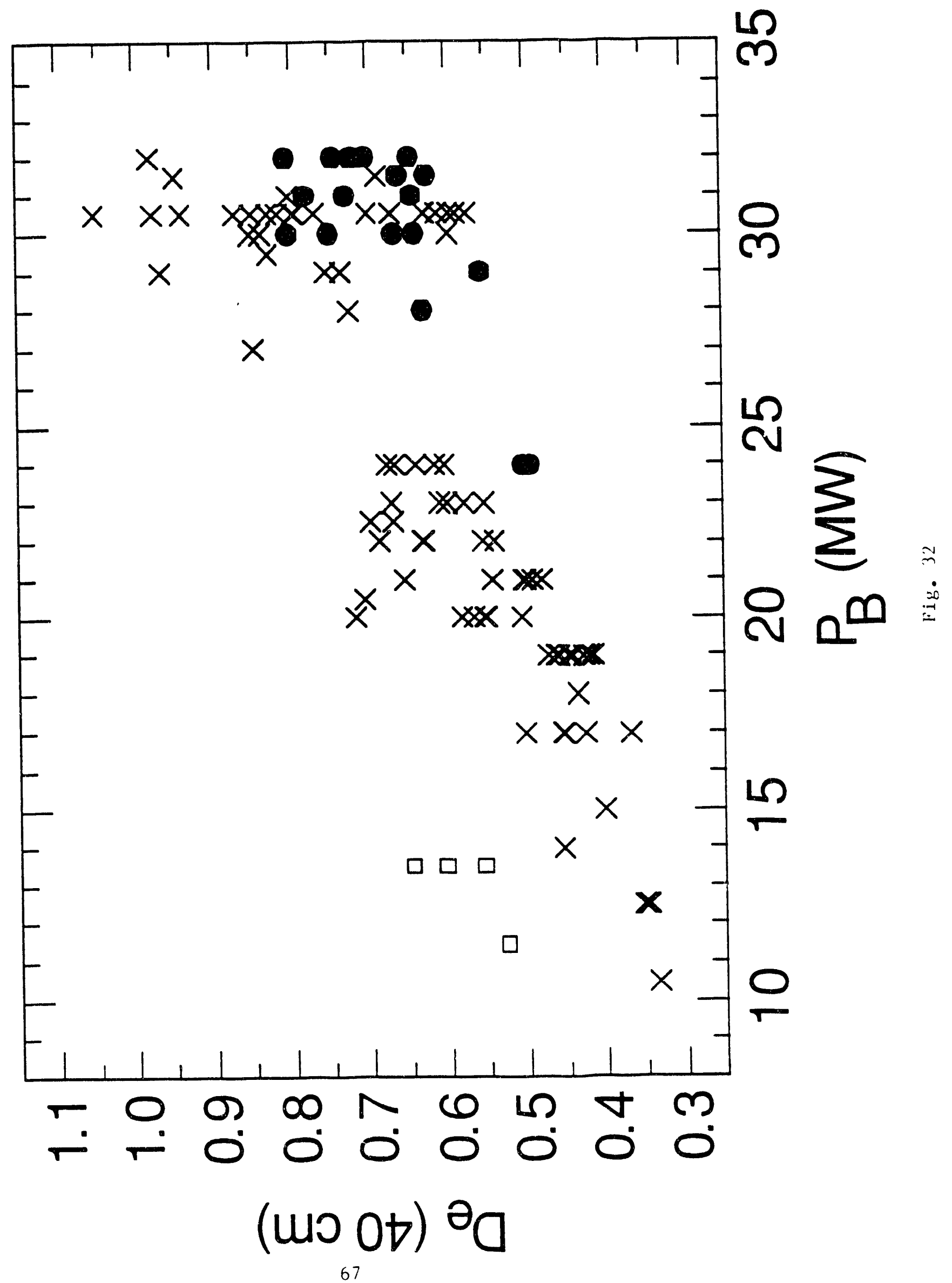


Dr. F. Peoboni, Univ. of Wottongong, AUSTRALIA

Prot. M.H. Brennan, Univ. of Sydnoy, AUSTRALIA

Plesma Research Lob., Australien Nat. Univ., AUSTRALIA

Prof. I.R. Jones. Flinders Univ, AUSTRALLA

Prot. F. Cap, Inst for Theoretical Physics, AUSTRIA

Prot. M. Hoincior, Instiut tor Theorelische Physik, AUSTRIA

Prot. M. Goossens, Astronomisch instiusur, BELGIUM

Ecole Royedo Mititire, Leb. do Phy. Plasmas, BELGIUM

Commission-Europoen, DG. XII-Fusion Prog., BELGIUM

Prof. R. Bouciaus, Rijksunivordibit Gent BELGIUM

D. P.H. Sakenake, Instituto Frica. BRWZIL

Instituto Neciond Do Pocquieas Especiais-INPE, BRUZIL

Documents Orios. Alomic Energy of Cenada Lid., CANADA

Dr. M.P. Bectrynskj, MPB Technologies, Inc., CANADA

Dr. H.M. Skarsoard, Univ. of Saskakthowen, CANADA

Prof. J. Toictmamn, Univ. of Montrod, CANAOA

Prot. S.R. Sreenivecan, Univ. of Calgary, CANADA

Prof. T.W. Joh ston, INRS-Energio, CANADA

Dr. R. Botton, Contre canedien de tusion magnátique, CANADA

Cx. C.R. James., Univ. of Nborm. CANADA

Dr. P. Lukéc, Komericketho Univorszita, CZECHOSLOVAKIA

The L branien, Culham Laborabry. ENGLAND

Litrary, R61, Ruthortord Apploton Laboratory. ENGLAND

Wrs S.A. Hurctinson, JET Librery, ENGLAND

Dr. S C. Sherma, Univ. of South Pacific. FIJI ISLANDS

$P$. Mehonen, Univ. of Helsinti, FINLAND

Prof. M.N. Buseac, Ecole Polybchnique,. FRANCE

C. Noutrot, La do Physique des Milieux bonises. FRANCE

J. Radat CENCADARACHE - Bat 506, FRANCE

Prol. E. Economow, Univ. of Creto, GREECE

Ms. C. Rinni, Univ. of loamina, GREECE

Or. T. Mud, Acadon iy Bitliographic Ser., HONG KONG

Preprnt Libray, Hungarian Acadamy of Sa., HUNGARY

Ci : . D: supe, Sitha inst of Nucter Pty sics, INDIf:

Ur. P. Kaw, Inst. to Plasma Research, INDIA

D. P. Rosenaw, Israel inst of Tertinotogy, ISPAEL

Libranan, Intomaional Conter for Theo Physics, ITALY

Miss C De Pab, Associazione EURATOMENEA, ITALY

D. G. Grosso, 180 tuto di Fisica der Plasma. ITALY

Prof G Rosiangni, Isturio Gas lonizzar Del Cnr, ITALY

Dr. H Yamaso. Tochiba Ros \& Dever Centor, JAPAN
Prot. I. Kawakemi, Hroshima Univ., JAPAN

Prot. K. Nishikawa, Hiroshima Univ., JAPAN

Director, Jepen Alomic Energy Research Inst. JAPAN

Prof. S. Itoh, Kyushu Univ.. JAPAN

Recearch Into. Cr., Nabional Instit for Fusion Science, JAPAN

Prof. S. Teneka, Kyoto Univ., JAPAN

Lorer; Kyoto Univ., JAPAN

Prot. N. Inow. Univ. of Tokyo, JAPAN

Secretary, Plesma Section, Eloctrolechnical Lab., JAPAN

S. Mori. Tectinical Advisor, LAERI, JAPAN

Dr. O. Miterai, Kumemoto Inst of Tectinotogy, JAPAN

J. Hycon-Sook, Korea Abmic Energy Pasearch Inst, KOREA

D.I. Chai, The Korea Adv. Inst of Sai. \& Tech., KOREA

Prot. B.S. Litoy, Univ. of Waikato, NEW ZEALAND

Inst of Phycica. Chineso Acad SCI PEOPLE'S REP. OF CHINA Lbrery, Inat of Plasma Physics, PEOPLE'S REP. OF CHINA Tainghu Unir. Library, PEOPLE'S REPUBLIC OF CHINA

$Z$ L.S.W. Inst Physics, PEOPLE'S REPUBUC OF CHINA

Prol. J.A.C. Cebred, Instituto Superior Tecrico, PORTUGAL

Dr. O. Potrus, Al I Cuza Univ., ROMania

Dr. J. Villiors. Fusion Studies, AEC. S. AFAICA

Prot. M.A. Hellborg, Univ. of Natal, S. AfricA

Prof. D.E. Km, Pohang Inst. of Sa. \& Tech., SO. KOREA

Prot. C.I.E.MA.T, Fusion Division Library, SPAIN

Dr. L Swonflo. Univ. Of UMEA, SWEDEN

Libray, Royal Inst. of Tectindogy, SWEDEN

Prof. H. Whatmeon, Chaimers Univ. of Tech. SWETJEN

Contro Phys. Des Plasmas, Ecole Polytoch, SWIiZERLAND

Biblioder, Inst. Voor Plasma-Fysica, THE NETHERLANDS

Asst Prot. Dr. S. Cekir, Midde East Tech. Univ., TURKEY

Dr. V.A. Guktikh, Sai. Ros. Inst Eloctophys.I Apparatus. USSR

Dr. D.D. Ayubr, Siberian Branch of Academy of Sa., USSA

D. GA. Elseev, I.V. Kurchator Inst, USSA

Librarian. The Ukr.SSR Academy of Scancos. USSR

Dr. LM. Kovizinmykh, Inst. of General Physics, USSA

Kormtorsctungsandage GmbH, Zentralbibliothek, W GERMAiv Bibliothok, Inst Fur Plasmatorschung. W GERMANY

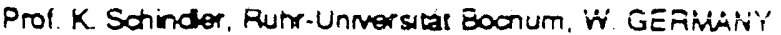
Dr. F Wagner, (ASOEX), Max-Plandx-Instuth, W. GERMANY Libranen, Max.Planck-Insour. W GERMANY

Prot. A.K Janew. Inst of Physics. YUGOSLAVIA 

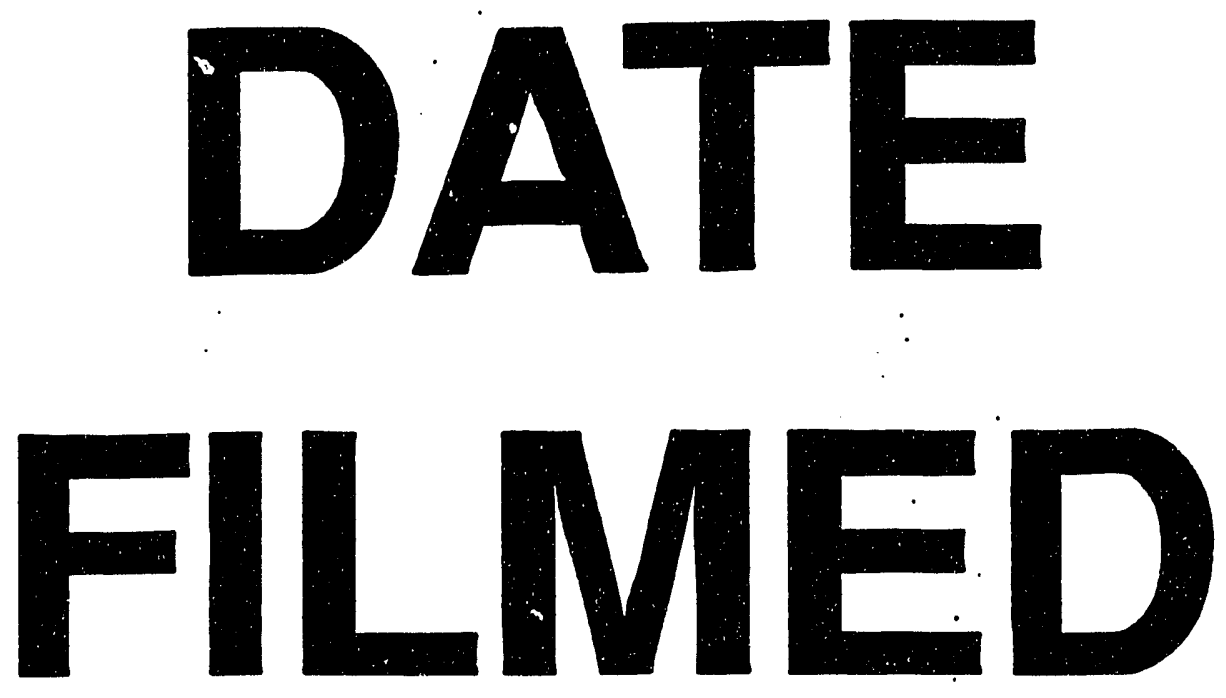

$10 / 20 / 93$
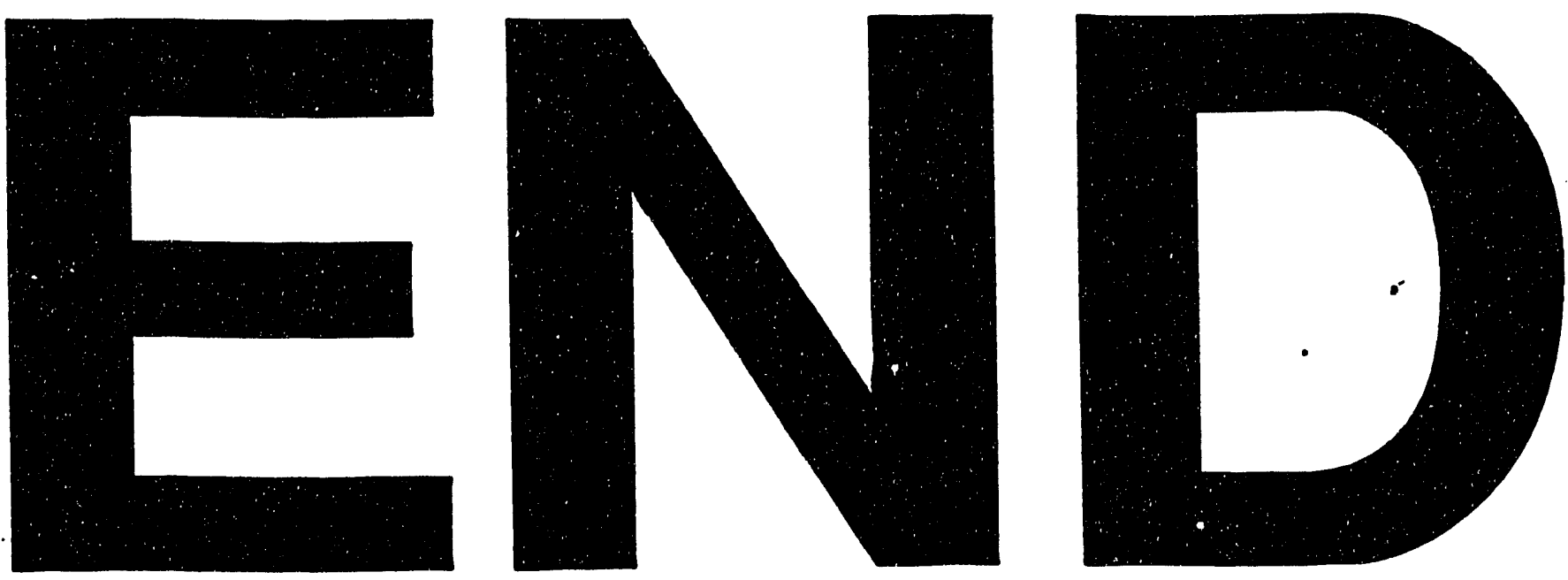
- " 